\title{
Digital-Image Processing and Image Analysis of Glacier Ice
}

Chapter 1 of

Section D, Digital-Image Processing

Book 7, Automated Data Processing and Computations

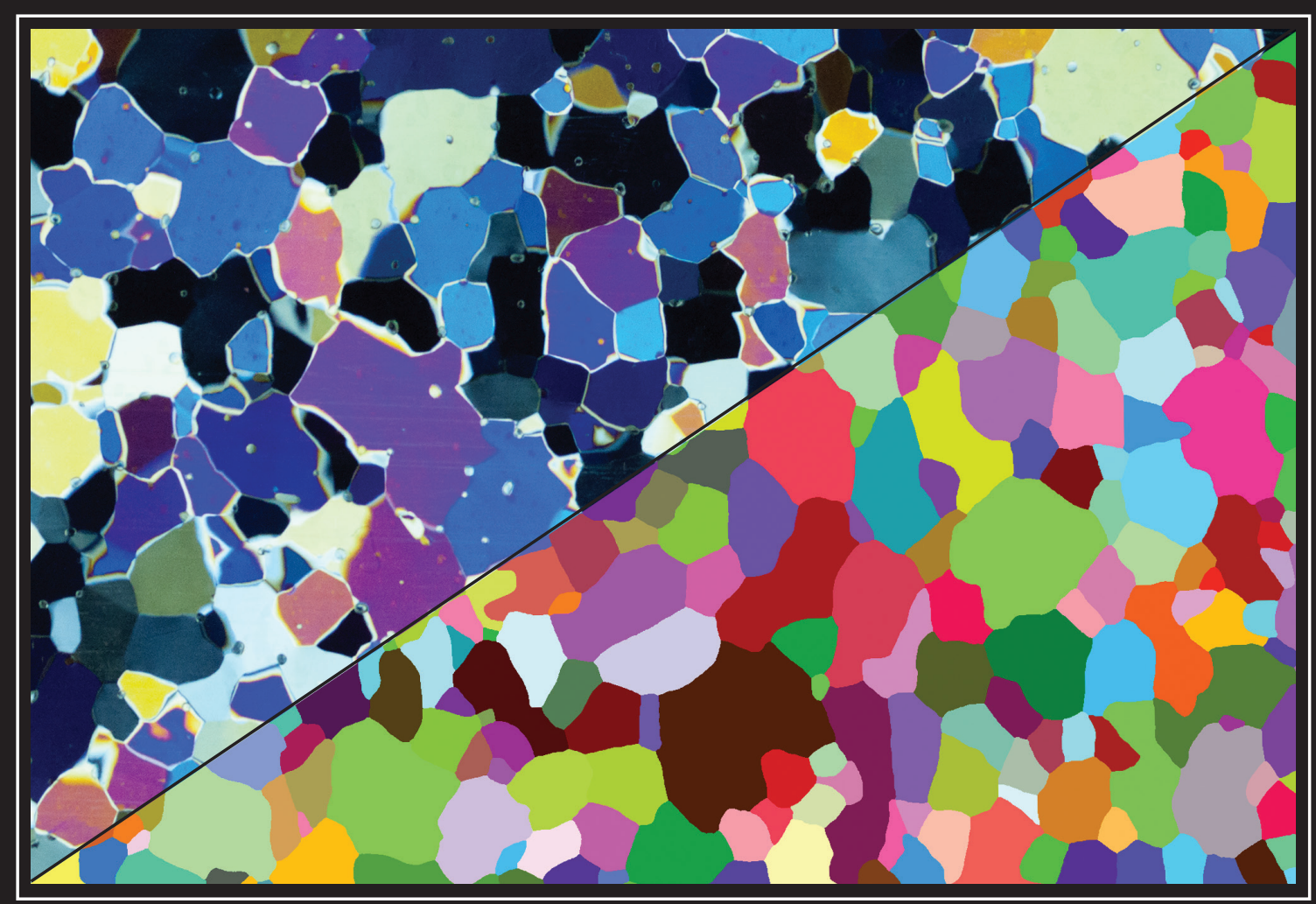

Techniques and Methods 7-D1

U.S. Department of the Interior U.S. Geological Survey 
Cover. Split view of the observed grain structure (upper left) of a sample from the main West Antarctic Ice Sheet (WAIS) Divide ice core (WDC-06A) and its contiguous image-processed representation (lower right) ready for ingestion in microstructural model. 


\section{Digital-Image Processing and Image Analysis of Glacier Ice}

By Joan J. Fitzpatrick

Chapter 1 of

Section D, Digital-Image Processing

Book 7, Automated Data Processing and Computations

Techniques and Methods 7-D1 


\title{
U.S. Department of the Interior SALLY JEWELL, Secretary
}

\section{U.S. Geological Survey Suzette M. Kimball, Acting Director}

\author{
U.S. Geological Survey, Reston, Virginia: 2013
}

For more information on the USGS - the Federal source for science about the Earth, its natural and living resources, natural hazards, and the environment, visit http://www.usgs.gov or call 1-888-ASK-USGS.

For an overview of USGS information products, including maps, imagery, and publications, visit http://www.usgs.gov/pubprod

To order this and other USGS information products, visit http://store.usgs.gov

Any use of trade, firm, or product names is for descriptive purposes only and does not imply endorsement by the U.S. Government.

Although this information product, for the most part, is in the public domain, it also may contain copyrighted materials as noted in the text. Permission to reproduce copyrighted items must be secured from the copyright owner.

Suggested citation:

Fitzpatrick, J.J., 2013, Digital-image processing and image analysis of glacier ice: U.S. Geological Survey Techniques and Methods, book 7, chap. D1, 21 p., http://dx.doi.org/10.3133/tm7D1.

ISSN 2328-7055 (online) 


\section{Contents}

Abstract

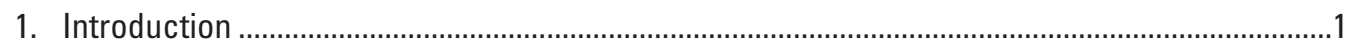

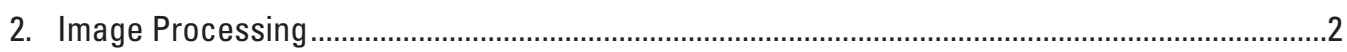

2.1 Sample Preparation and Digital Photography of Thin Sections.........................................2

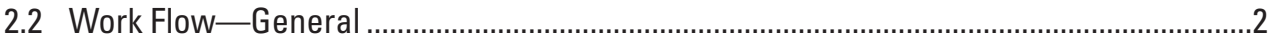

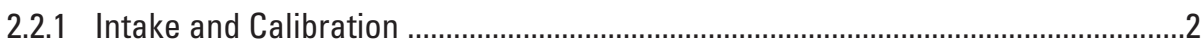

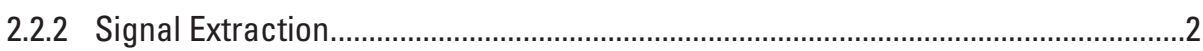

2.3 Intake and Calibration............................................................................................

2.3.1 Compositing Multiple-Frame Images ...................................................................

2.3.2 Spatial Calibration ...........................................................................................

2.3.2a Spatial Calibration in Photoshop....................................................................

2.3.2b Spatial Calibration in FoveaPro...................................................................

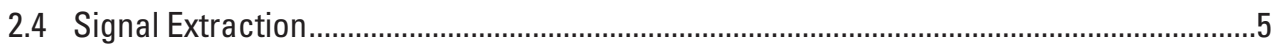

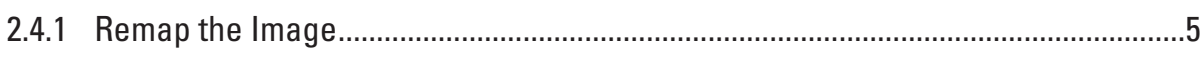

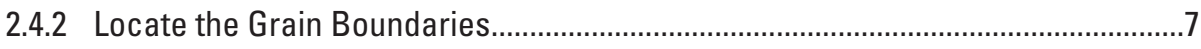

2.4.3 Threshold (Binarize) the Grain-Boundary Edge Image .............................................7

2.4.4 Reject Unwanted Features and Clean Up Edges .................................................7

2.4.5 Improve Continuity of the Grain-Boundary Mesh ......................................................7

2.4.6 Check and Correct the Coarse Grain-Boundary Mesh ..........................................8

2.4.7 Skeletonize the Corrected Grain-Boundary Mesh.................................................8

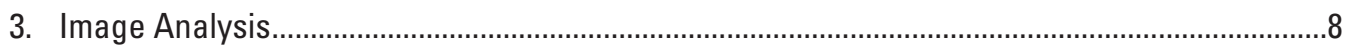

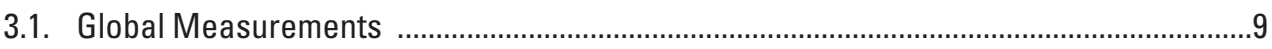

3.1.1 Mean Planimetric Grain Area........................................................................

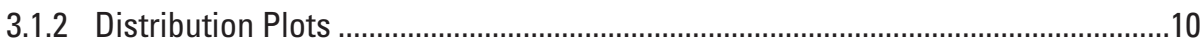

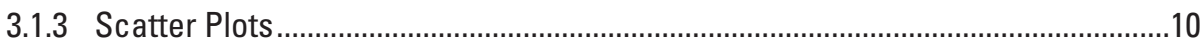

3.1.4 Cluster Analysis .......................................................................................... 10

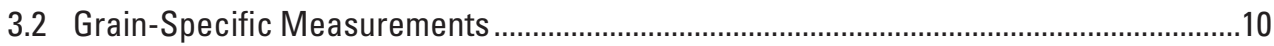

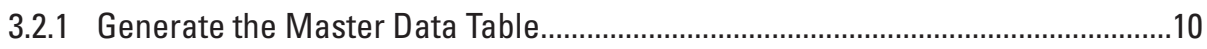

3.2.2 Statistical Measurements...............................................................................12

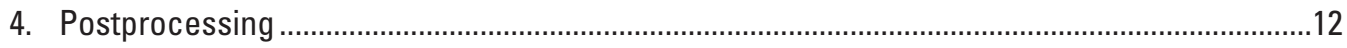

4.1 Example 1. Mean Grain Size as a General Guide........................................................12

4.2 Example 2. Population Statistics and Distributions ........................................................12

4.3 Example 3. Ice Behavior Compared ........................................................................ 14

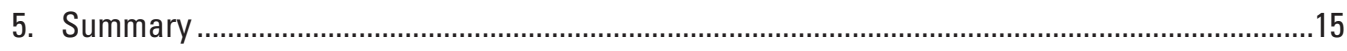

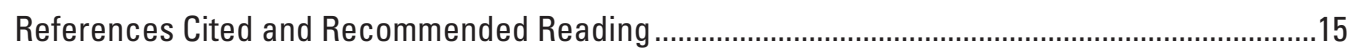

Appendix 1. Image-Processing Walk Through ....................................................................19

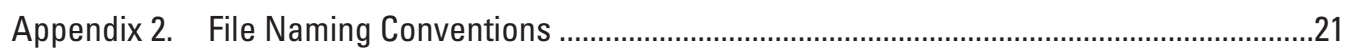




\title{
Figures
}

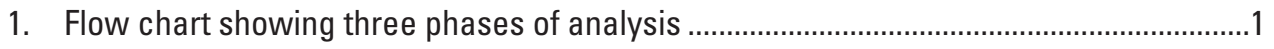

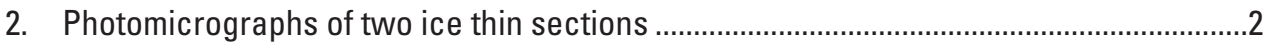

3. Screen shot of a Photoshop "Photomerge" options window ............................................

4. Screen shot of a FoveaPro spatial calibration selection window and calibration window

5. Raster images of start and end points for image-processing phase of grain analysis

6. Screen shot of layer-naming conventions in Photoshop for a typical edge stack

7. Screen shot of a FoveaPro typical covariance matrix for the principalcomponent-analysis transform process

8. Photomicrograph of an ice thin section showing image transformations

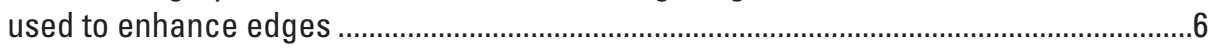

9. Screen shots showing determination of mean planimetric grain size in Photoshop.

10. Bar diagram of a FoveaPro distribution plot based on equivalent grain diameter

11. Scatter diagram in FoveaPro showing ice-grain elongation on $\mathrm{x}$-axis and area on y-axis

12. Cluster-analysis diagram in FoveaPro showing elongation and alignment.....................11

13. Screen shot of a StatPlus descriptive statistics window ............................................13

14. Diagram of mean ice-grain size compared with depth in the West Antarctic

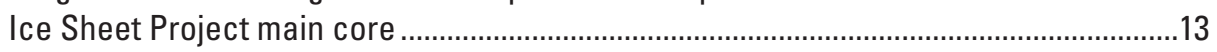

15. Diagram of two grain-area populations from the West Antarctic Ice Sheet Divide core

16. Diagram of rates of recrystallization in "normal" ice-grain growth regimes based on several ice-core sites in Antarctica and Greenland.....

\section{Abbreviations Used in This Report}

\author{
$\mathrm{cm}$ centimeter \\ m meter \\ ACR Antarctic Cold Reversal \\ PCA principal components analysis \\ RGB red, green, blue
}




\title{
Digital-Image Processing and Image Analysis of Glacier Ice
}

\author{
by Joan J. Fitzpatrick
}

\section{Abstract}

This document provides a methodology for extracting grain statistics from 8-bit color and grayscale images of thin sections of glacier ice - a subset of physical properties measurements typically performed on ice cores. This type of analysis is most commonly used to characterize the evolution of ice-crystal size, shape, and intercrystalline spatial relations within a large body of ice sampled by deep ice-coring projects from which paleoclimate records will be developed. However, such information is equally useful for investigating the stress state and physical responses of ice to stresses within a glacier. The methods of analysis presented here go hand-in-hand with the analysis of ice fabrics (aggregate crystal orientations) and, when combined with fabric analysis, provide a powerful method for investigating the dynamic recrystallization and deformation behaviors of bodies of ice in motion.

The procedures described in this document compose a step-by-step handbook for a specific image acquisition and data reduction system built in support of U.S. Geological Survey ice analysis projects, but the general methodology can be used with any combination of image processing and analysis software. The specific approaches in this document use the FoveaPro 4 plug-in toolset to Adobe Photoshop CS5 Extended but it can be carried out equally well, though somewhat less conveniently, with software such as the image processing toolbox in MATLAB, Image-Pro Plus, or ImageJ.

\section{Introduction}

The reconstruction of paleoclimate records from ice cores requires (1) a multiplicity of chemical, isotopic, and electrical measurements which are then related to a depthage framework that assumes continuous deposition of snow at the glacier surface in the past, and (2) that the geological principles of superposition and original horizontality apply, unless proven otherwise. The physical properties of an ice core can be of great value in evaluation of the "proven otherwise" assumption. The scientist who examines the visual stratigraphy of an ice core, and thin sections made from it, is commonly the first one to detect signs of trouble with this assumption. By alerting other scientists working on the same core to specific depths at which discontinuities in the depthage relation in the core may be present, the physical properties specialist can prevent the misinterpretation of artifacts due to ice deformation as climate transitions.
Beyond the utility of physical properties measurements to the paleoclimate- reconstruction community, the characterization of grain growth and recrystallization behaviors can also inform ice sheet modeling efforts, because the large-scale deformation behavior of an ice body depends on the accommodation of stress at the microscopic scale. Careful characterization of the evolution of grain size and shape distributions and nearest-neighbor relations ("texture"), coupled with observation of fabrics and microstructures revealed in thin section, provides direct evidence of the mechanisms of dynamic recrystallization operating within specific depth intervals in the ice body. These mechanisms ultimately control flow-law behavior.

The analysis of thin sections from an ice core proceeds in three separate phases: image processing, image analysis, and postprocessing (fig. 1). These three phases are described in this document, along with specific inputs and outputs. There are many intermediate steps in this process, and maintaining
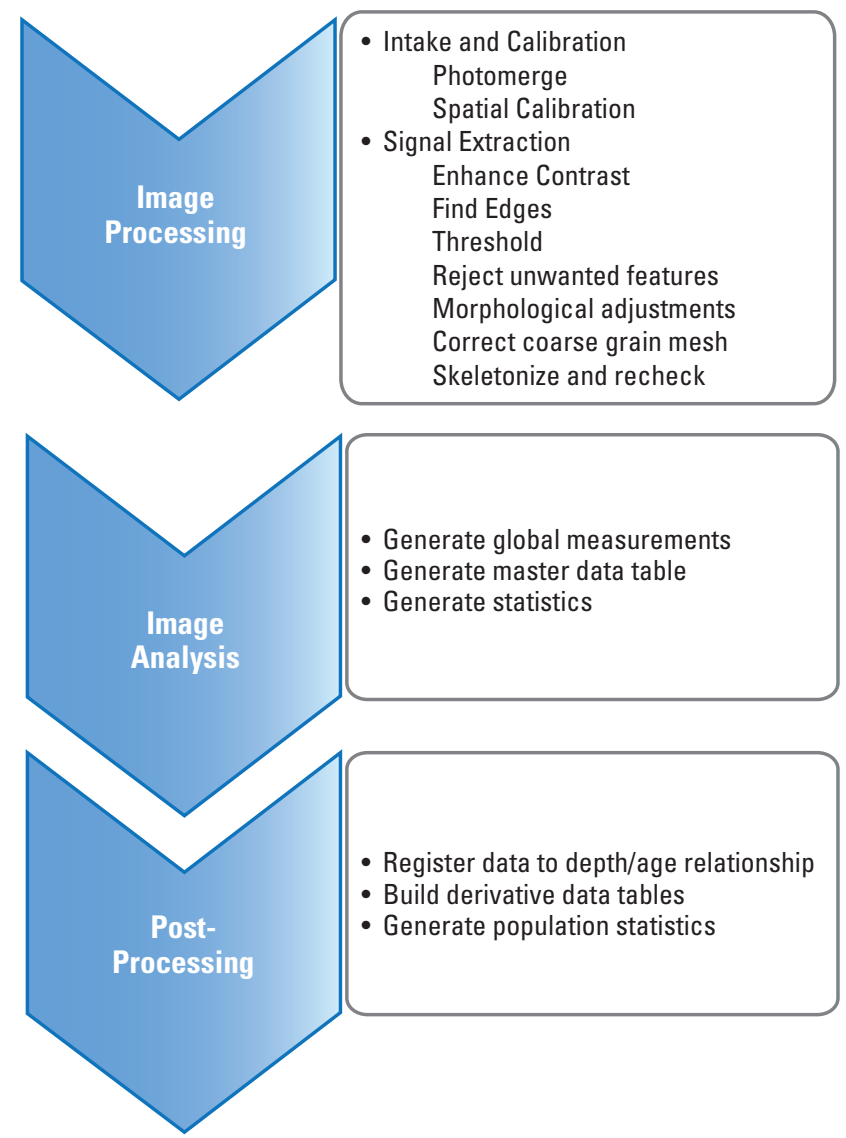

Figure 1. Analytical flow chart of the three phases of analysis. 
consistent file structures and file naming conventions is imperative. Examples of images are provided for each step in the processing and analysis (appendix 1) and of file-naming conventions (appendix 2). For the sake of clarity, throughout the rest of this document Photoshop CS5 Extended commands and menu choices are indicated in blue and FoveaPro commands and menu choices are indicated in red.

\section{Image Processing}

\subsection{Sample Preparation and Digital Photography of Thin Sections}

The starting place for these analyses is the digital image of the thin section. A description for preparing these thin sections from an ice core is beyond the scope of this document, but some special conditions apply to ice thin sections and images prepared from them for grain analysis, and these will be mentioned here.

To optimize samples for image analysis, use thin sections that are thinned to display nothing higher than low secondorder interference colors (fig. $2 A$ ). Sections that produce high second-order or third-order colors will display geometrically complex grain boundaries with multiple birefringence minima that can be difficult to interpret (fig. $2 B$ ). Bear in mind that as work proceeds down the core, it may become necessary to adjust the size of the thin section or the sampling interval to ensure that a sufficient number of grains is available for the analysis to be statistically meaningful.

For high-resolution work, it is usually necessary to take multiple frames of an individual thin section and to merge the frames into a single image file at the beginning of processing. One of the frames must contain the image of a ruler or other scale mark to facilitate the direct measurement of a scale factor.

Photograph samples in at least two optical orientations to avoid ambiguity when grain boundaries in extinct grains are located. Multiple images can be superimposed in adjacent layers in Photoshop at the beginning of the analysis to facilitate comparison of the appearance of individual grains under variable extinction conditions.

\subsection{Work Flow-General}

Processing the image to prepare it for analysis and postanalysis proceeds in two steps.

\subsubsection{Intake and Calibration}

These steps assemble the image and provide a spatial calibration. They result in a layered composite red-green-blue (RGB) image (if the image is a composite of several frames) and an optimized, flat RGB image that is spatially calibrated both in Photoshop and in FoveaPro.
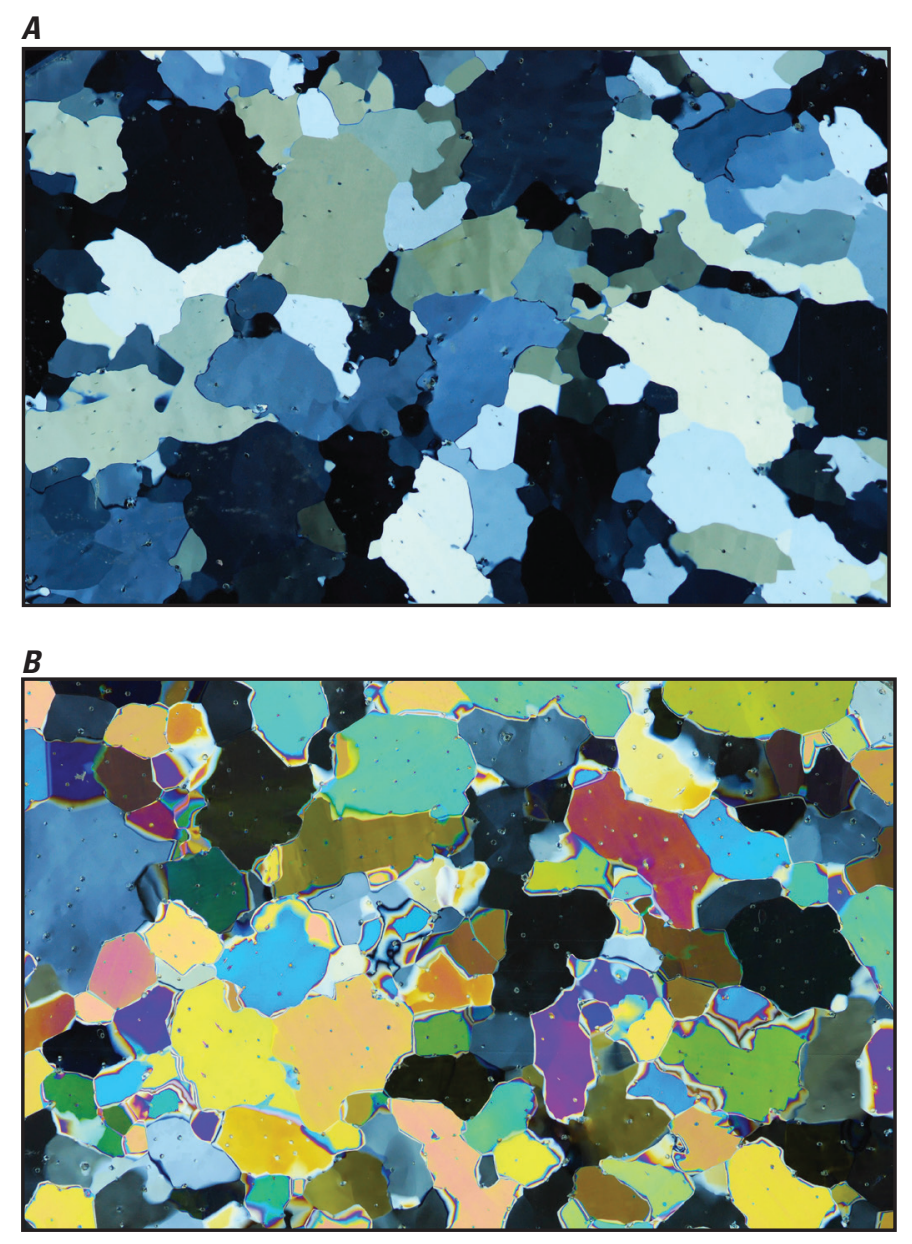

Figure 2. Two photomicrographs of ice thin sections; crosspolarized light. $A$, Thickness optimized for grain analysis at about $120-140$ micrometers. $B$, Thin section is too thick, making it difficult to locate grain boundaries.

\subsubsection{Signal Extraction}

These steps use image-processing algorithms to separate the target spatial features (for example, grain boundaries or bubbles) from the rest of the information in the image. In grain images, these steps produce two results:

- a multilayered red, green, blue (RGB) image (hereafter referred to as the "RGB stack") that contains, at the least, the two aligned starting RGB images, the coarse grain-boundary mesh, and the final, fine grain-boundary (1 pixel) mesh used for analysis; and

- a multilayer grayscale image file (hereafter referred to as the "edge stack") containing, at the least, the starting principal components analysis forward transform of the RGB image, or a similar contrast-maximized treatment of the RGB image; the result of the edge-finding algorithm; the thresholded edge image; and the final coarse and fine edge grain-boundary meshes. 
It is important to develop file-naming conventions (see appendix 2 for recommended file-naming conventions) and directory structures that are meaningful and that will permit the analyst to maintain consistency and to easily navigate the many intermediate files and images that will be produced during the processing and analysis procedures. At a minimum, maintain separate directories for the original image frames and composites built from them, for the RGB and grayscale image stacks that will be created, and for the analytical results that will be generated for each image of each sample.

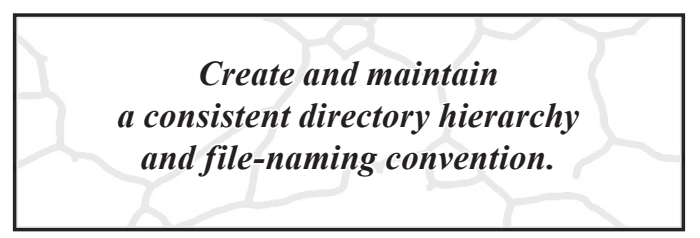

It is also important to use every opportunity to check the accuracy of your work. Several procedural checks are built into the process described in this document, but analysts should exploit every additional opportunity to check the reasonability of their results at every step of the analysis.

\subsection{Intake and Calibration}

\subsubsection{Compositing Multiple-Frame Images}

For an individual thin section, the image of the entire section may need to be composited from multiple highresolution frames, at least one of which contains a scale or marker in the frame. (A typical 4" $\times 4$ " section requires nine frames at a scale factor of 800 pixels per centimeter $(\mathrm{cm})$.)
To create the composite image, choose File $>$ Automate $>$ Photomerge from the topmost menu bar in Photoshop, select "Collage" and "Blend Images Together" and browse to the directory containing all of the frames required for the composite image. Load all the required frames into the source file frame and select "OK" (fig. 3). You will be able to follow the progress of the compositing process in the Layers Panel that will open automatically.

- Once the composited image has been assembled, save it with its layer structure intact (see appendix 2 for filenaming conventions). You may need to reexamine the masking and blending that take place during compositing at a later time.

- File output: full-layered composite RGB image

- Flatten (reduce to a single layer) the image, rename it, and save it as your starting RGB file. (Photoshop layers panel controls $>$ flatten)

- File output: flattened composite RGB image

Complete these steps for all images of the thin section photographed in different optical orientations. Create a new RGB file with the flattened images registered to each other, each in their own layer. Using Photoshop $>$ File $>$ File Info, enter the sample designation and analyst's name into the "Document Title" and "Author" fields. Into the "Keywords" field, enter the true depth range, spatial calibration value (see section 2.2.2), and the numeric range of the individual raw image files used to create the photomerged image. If the image being processed has been acquired on a vertical sample, indicate which direction is stratigraphic "up." Name and save this file. It is the base for the RGB stack file.

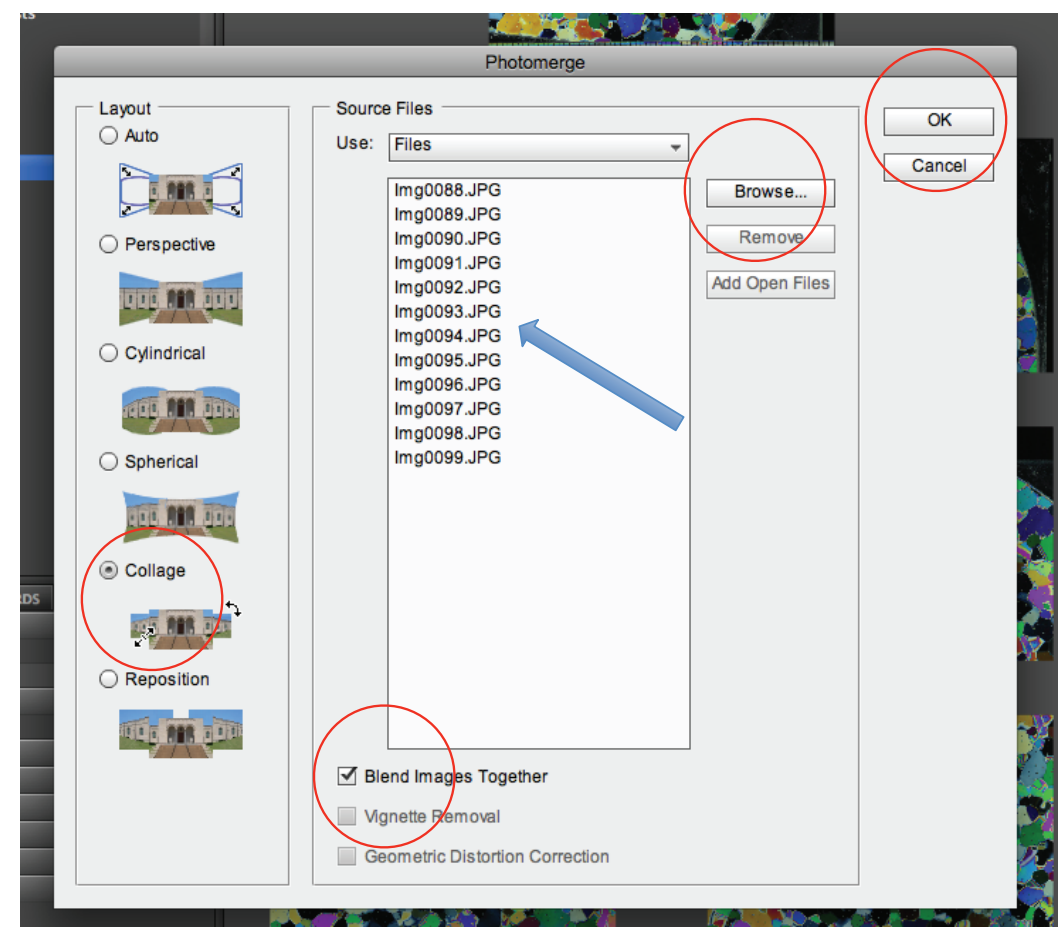

Figure 3. Photoshop CS5 Extended, "Photomerge" options window. 


\subsubsection{Spatial Calibration}

In order to convert measurements made on the digital image to real-world units, a scale factor must be derived that relates a number of pixels in the image to a real-world length. The image of the ruler embedded in the digital photo enables us to accomplish this.

\subsection{2a Spatial Calibration in Photoshop}

The "Set Measurement Scale" option in Photoshop CS5 Extended provides a useful utility for performing direct on-screen measurements on the fly. Although it's not necessary to perform this calibration (FoveaPro uses only its own spatial-calibration file), it provides some useful conveniences and a quick cross-check with the spatial calibration provided within FoveaPro.

Starting with the image in the RGB stack that shows the scale or marker, increase the screen magnification to ensure that the image of the scale is large and easy to read. From the Photoshop CS5 Extended menu select Analysis $>$ Set Measurement Scale $>$ Custom. In the "Options" window, set the "Logical Length" value to 1 and the "Logical Units" to "cm" and, using the Photoshop ruler tool, draw a line $1 \mathrm{~cm}$ in length along the image of the ruler in the RGB image. Take note of the value that appears in the pixel window of the > "Set Measurement Scale" options window when you've performed this step. This number will be used to cross-check the FoveaPro spatial calibration result in the next step, and it can be used to have Photoshop place a scale marker on the original flat composite image for publication purposes.

\section{A}

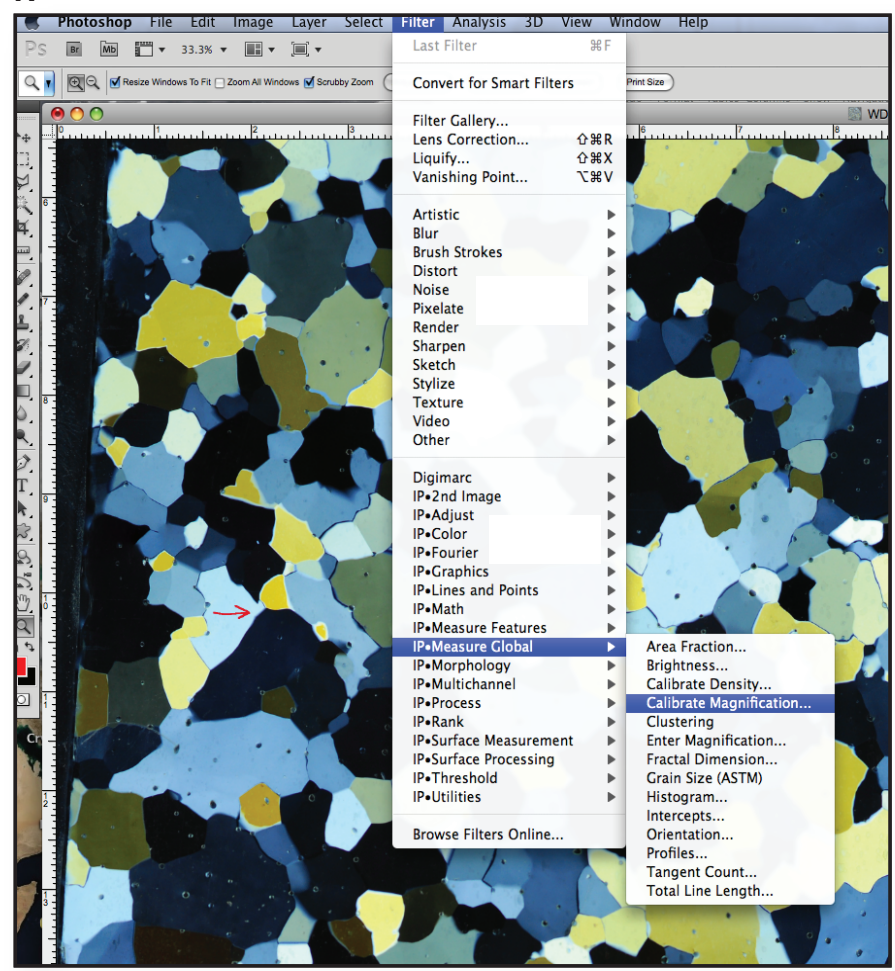

\subsection{2b Spatial Calibration in FoveaPro}

With the base layer of the RGB stack selected, choose Filter $>$ Measure Global $>$ Calibrate Magnification (fig. 4A). Note that the FoveaPro filters appear below the Photoshop filters in the drop down menu. The FoveaPro calibration window will open.

The default starting location of the FoveaPro "Calibrate Magnification" window (fig. $4 B$ ) is the upper left-hand corner of the image. Use the sliders in this window to locate the portion of the image containing the ruler. You cannot control the magnification of this window. Ensure that the "cm" radio button is checked and that a value of 1.0 is entered into the "Value" window. Click on a long tick (centimeter mark) on the image of the ruler, scroll to a ruler tick $1 \mathrm{~cm}$ away, and place the second mark. These two marks will cause the pixel value under the image of the ruler to update to the number of pixels between your two marks. If the values determined in both Photoshop and FoveaPro are within 0.5 percent of each other, select the "Save As" button from this window and name the file using the appropriate name convention. If the two values differ by more than 0.5 percent, perform the calibration operations again until the two values agree to within this tolerance.

As you navigate through the course of the image processing and analysis, be mindful of ensuring that your spatial calibration applies to the image you are working on. Both Photoshop and FoveaPro retain the last-used value. If you are returning to an image after working on another that you calibrated more recently, you will need to read in the correct calibration file for the image you are currently working on ("Import" button in fig. 4B).

\section{B}

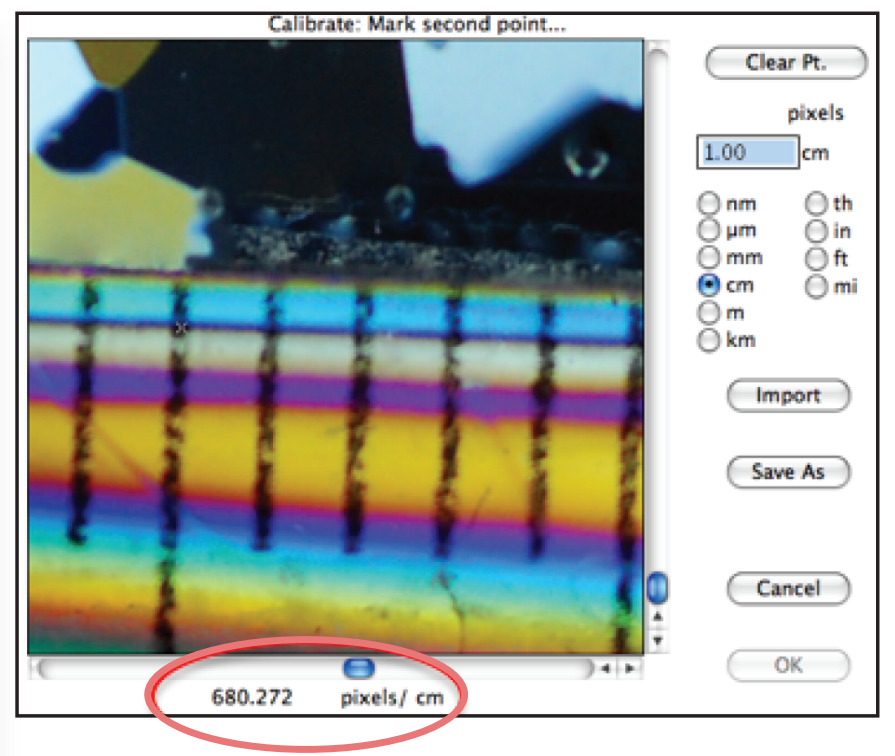

Figure 4. FoveaPro, spatial calibration selection $(A)$ and calibration $(B)$ windows. When two end points are identified in the calibration window, the calibration value (red ellipse) will update. 


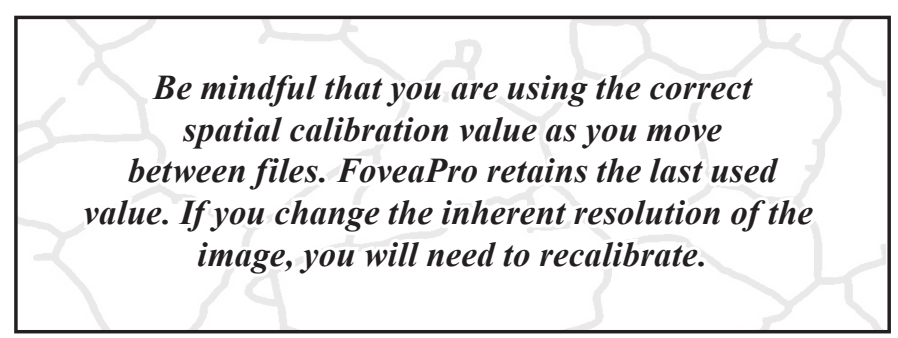

\subsection{Signal Extraction}

This section describes the steps taken to separate the features in the image you want to analyze (signal) from everything else (background).

The image of a thin section is an irregular tessellation. The goal of this phase of the processing is to identify and separate a continuous, closed line (mesh) that represents the location of grain boundaries in the image (fig. 5). In order to do this, we exploit the color or contrast changes at the grain boundaries.

There are many ways to accomplish each step in this process. Depending on the characteristics of the starting image, some contrast-enhancement and edge-finding algorithms may prove to be more successful than others. The algorithms suggested here have proven to be successful in most instances.

$\boldsymbol{A}$
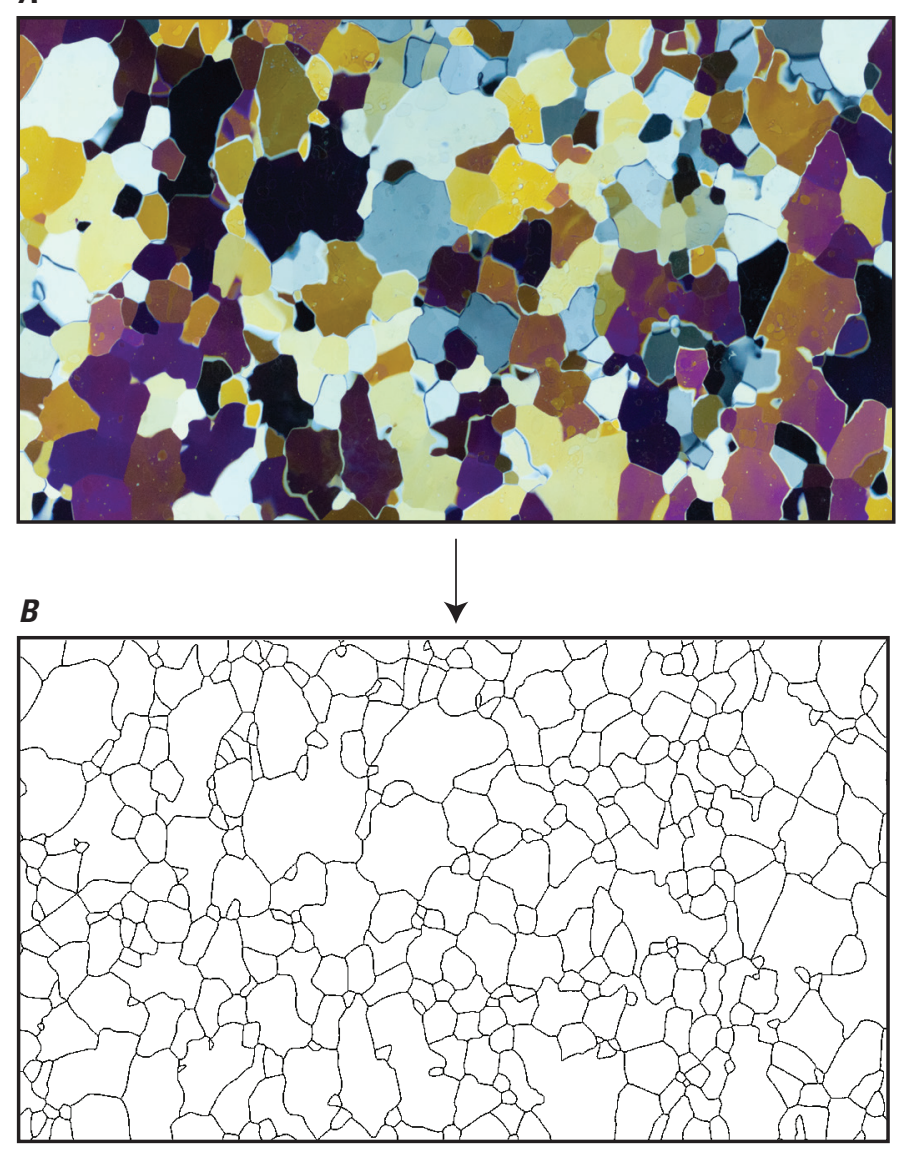

Figure 5. Starting and ending points for image-processing phase of grain analysis. $A$, Starting RGB (red-green-blue) digital image; $B$, Final grain-boundary map derived from processing of the RGB image.
The image processing steps needed to accomplish the isolation of the grain boundaries typically take the following operations in order:

1. Remap the image to its best possible contrast

2. Locate the edges

3. Threshold (reduce from grayscale to black and white) the edge file

4. Reject unwanted features (such as bubbles), clean up the edges

5. Use morphological operators to improve the continuity of the edge mesh

6. Superimpose the edge mesh back onto the RGB image in the RGB stack file, check it and, if needed, correct it

7. Skeletonize the corrected mesh.

The black and white, skeletonized (reduced to 1-pixel width) edge mesh resulting from the last step (7) is the input into the analytical routines used in the next phase. A visual walk through of each of these steps is provided in appendix 1 of this document.

Staying organized: To keep track of how you perform the steps in this phase, create a new layer in the edge stack for each processing step and name the layer with the algorithm used. For example, if you located edges by using the Photoshop "Find Edges" function on the RGB image, name the output layer "Photoshop RGB edges." When you're ready to move to the next image-processing step, duplicate the layer you will use as input and use the next operator on the duplicate layer, then rename it. In a typical analytical sequence in an edge stack file (fig. 6), each layer resulted from an image-processing operation on the layer below it.

\subsubsection{Remap the Image}

The purpose of this step is to provide the best possible input for the edge-finding algorithm that will be used in the next step. To do this we remap the starting RGB image using principal components analysis.

- Open the RGB stack file you created earlier (section 2.2.2). If you have more than one image of your sample thin section recorded in multiple orientations, ensure that they are loaded into the RGB stack as unique layers that are aligned with each other. A typical RGB stack file will have two stacked images at this point.

- Choose the stacked RGB image with the best color contrast and duplicate it into a new layer. Name the new layer "principal component analysis result."

- With this new layer selected, choose Filter $>$ IP•Multichannel $>$ Compute principal components analysis (PCA) transform; a pop-up window will display the covariance matrix resulting from the computation of the eigenmatrix. Most of the significance will reside in 


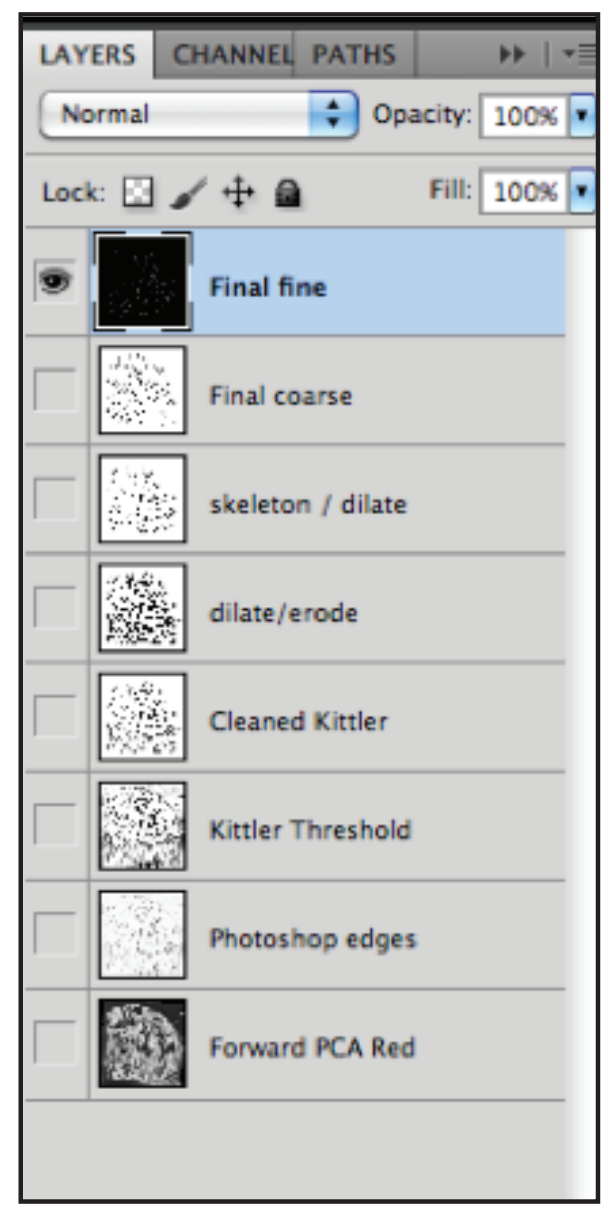

Figure 6. Layer-naming conventions for a typical edge stack. Each layer is the result of an image-processing operation on the layer below it.

one row (fig. 7). If it does not, then a different approach may be required. For example, the starting image can be remapped into a different color space or this step can be omitted completely and the built-in Photoshop RGB edge-finding algorithm can be attempted directly on the RGB image (Photoshop $>$ Filter $>$ Stylize $>$ Find Edges).

- The next FoveaPro pop-up window will give you the opportunity to name and save the eigenmatrix file. Don't change the extension when you select a name, and save it to a location where you can easily find itthe next step is going to ask you to point to it.

\begin{tabular}{|cccc|}
\hline \multicolumn{4}{|c|}{ Eigenvalues and eigenvectors for color channels } \\
\hline Significance & Red & Green & Blue \\
\hline $65.4618 \%$ & 0.67948 & 0.73106 & -0.06211 \\
$29.0070 \%$ & -0.02635 & 0.10892 & 0.99370 \\
$5.53125 \%$ & 0.73322 & -0.67357 & 0.09327 \\
\hline
\end{tabular}

Figure 7. Typical covariance matrix for the principal-componentanalysis transform process. Most of the significance should reside in one row (red ellipse).
- Select Filter $>$ IP•Multichannel $>$ Apply PCA (forward). Point to the eigenmatrix file created in the previous step when queried. This step remaps the image using the transformation eigenmatrix. The remapped image with the largest dynamic contrast is mapped into the first ("red") channel in this image.

- With only the "PCA result" layer selected, switch to the "Channels" tab in Photoshop. Select the "red" channel only, copy it to the clipboard, and create a new file in Photoshop with this layer (Photoshop $>$ File $>$ New). Be sure to set the color space to grayscale. Photoshop will automatically size the new file to match the clipboard contents. The file you have just created will become your edge-stack file. Save and name it. The resulting image is the starting point for the edge-finding algorithm (fig. 8).

A

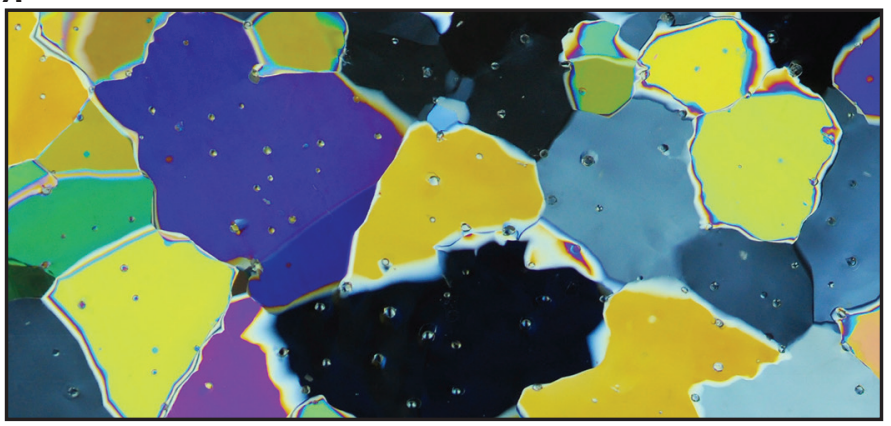

B

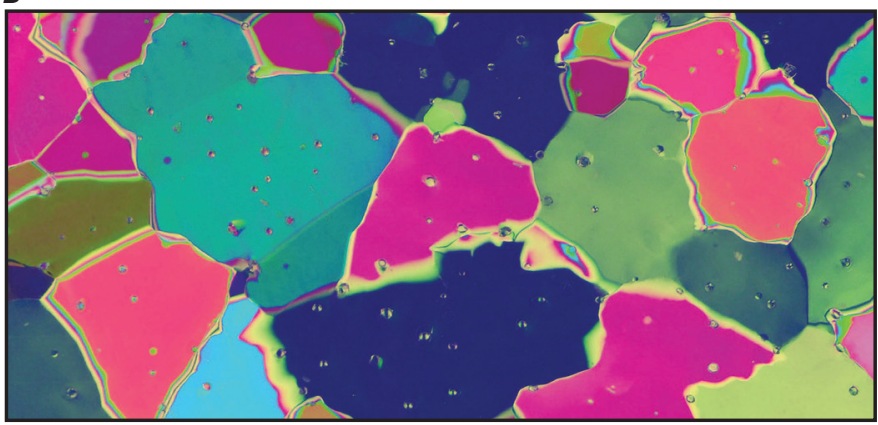

C

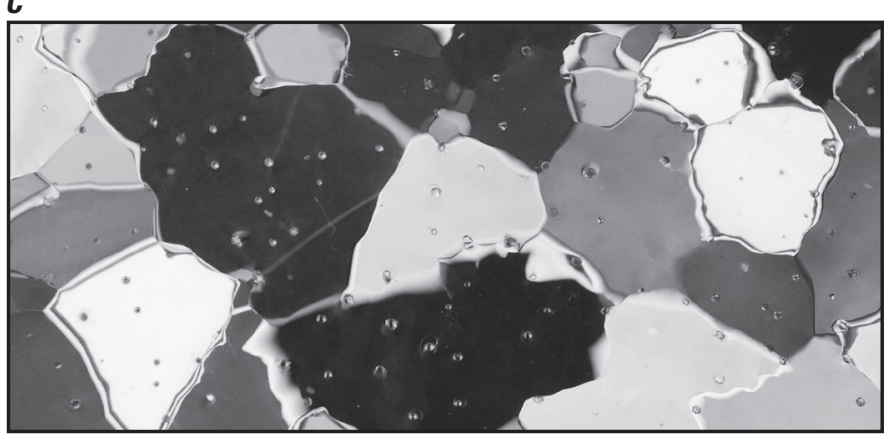

Figure 8. Progression of image transformations used to enhance edges in preparation for applying an edge-finding algorithm.

$A$, Original red-green-blue image. $B$, Image after principal-componentanalysis forward operation. $C$, Extraction from $B$ of channel with the most comprehensive number of grayscale values. $C$ represents the best possible contrast that can be achieved from the original image. 
This process can be repeated for each individual RGB image that has been recorded, and the result of the edgefinding algorithms can be Boolean added together at the end of the image-processing phase of analysis.

\subsubsection{Locate the Grain Boundaries}

- Duplicate the principal component analysis result into a new layer above the original (Layer panel controls $>$ duplicate layer)

- With the new layer selected, apply Filter $>I P \bullet$ Process $>$ Find Edges. This step will open a FoveaPro pop-up preview window that offers a selection of edge-finding algorithms of which Sobel, Kirsch, and Gradient Vector are probably the most useful. The window sliders and magnification controls can be used to move around inside the preview window to provide the analyst with an overview of how successful each algorithm is at producing the most complete grain boundary image with the least extraneous information. When the best operator is identified, apply it and rename the layer with the name of the operator used.

\begin{tabular}{|c|}
\hline Things to bear in mind when choosing \\
an edge-detection algorithm \\
- small isolated features can be filtered \\
out at a later point \\
holes or gaps can be filled in later using \\
morphological operators \\
too much detail is usually harder to fix \\
than too little
\end{tabular}

\subsubsection{Threshold (Binarize) the Grain- Boundary Edge Image}

This step creates a binary (black and white) rendering of the edge image in the active layer.

- Duplicate the "Find Edges" result and create a new layer with it (layer panel controls>duplicate layer)

- With the new layer selected, apply Filter $>I P \bullet T h r e s h o l d$ $>$ Bilevel Thresholding. A pop-up window with the bilevel-threshholding controls will open. You are given several options for setting a grayscale threshold point (value). Assuming that you are working with a white (255) background, every point in the image whose value is greater than the threshold value will be set to 255 (white). Every point below the selected threshold value is set to 0 (black). The preview window highlights features in the image that will be set to black in the chosen highlight color (default is red). Select the method or value that retains as much of the grain boundary as possible without retaining extraneous detail. A threshold value of 225 to 230 is usually successful. When you have chosen, select "OK" and rename the layer (in the layers panel) with the name of the chosen method or threshold value. (For example, in fig. 6, the Kittler algorithm was used to create the thresholded image that resides in the third layer above the Forward PCA base image.) This layer is the starting point for the image of the grain boundaries.

\subsubsection{Reject Unwanted Features and Clean Up Edges}

This step improves the appearance of the grain boundaries and allows you to discriminate out unwanted features

- Duplicate the thresholded image into a new layer and rename it.

- Using Filter $>I P \cdot M e a s u r e$ Features $>$ Reject Features, reject small unwanted features. Set a value starting at about 1,000 pixels to begin with and ensure that the foreground color in Photoshop is black. You may need to perform this step in subareas if your image is large. If needed, use the Photoshop rectangular marquee tool to select subareas and perform this clean up stepwise.

- Using the Photoshop tools, open (paint over with white) the outer edge of the grain-boundary mesh. You will not count grains that touch the edge of the thin section.

\subsubsection{Improve Continuity of the Grain- Boundary Mesh}

Depending on the quality of the grain boundary mesh at this point, you may or may not need to improve its continuity by using these operators.

- Duplicate the cleaned up image into a new layer and rename it.

- Dilate the mesh using the FoveaPro morphological operators (Filter $>$ IP•Morphology $>$ Classic or EDM Morphology), so that it becomes contiguous everywhere but at its edges (dilate to 11 or 12). Check the mesh against the principal component analysis image at the bottom of the stack for any gross discrepancies.

- Invoke Filter $>$ IP•Morphology $>$ Skeletonize. Follow this with Filter $>I P \cdot$ Morphology $>$ EDM Morphology and dilate the image to produce a clean, contiguous mesh (dilate to 6.50). This mesh is the starting coarse grain-boundary mesh. Name this layer "Starting Coarse Mesh." 


\subsubsection{Check and Correct the Coarse Grain-Boundary Mesh}

In this step you will superimpose the edge mesh back onto the RGB image in the RGB stack file and, if needed, correct it. Starting with this step, you will be moving between the edge-stack file and the RGB-stack file, so have both stacks open in Photoshop.

- Working in the starting coarse grain-boundary layer in the edge stack, use the selection tool in Photoshop with the color tolerance set to 1 and select an area in the background (white).

- Invoke Photoshop>Select>Inverse and copy this selection to the clipboard.

- Paste the coarse grain-boundary mesh from the clipboard into a new layer at the top the RGB stack. With the grain-boundary mesh still selected, remap the color to a bright orange or yellow by selecting a bright color from the Photoshop color palette and selecting Edit $>$ Fill $>$ Foreground color

- Working in the RGB stack, turn off any layers between the coarse grain-boundary layer you just created and the base RGB image from which you created the principal component analysis. If you need to reposition the mesh to coincide with this bottom-most layer, do so by selecting the layer and using the Photoshop Move Tool (not the hand tool, which will move the entire stack and not just the layer).

- Lock the base RGB image layer and verify that you're working in the coarse grain-boundary mesh layer. Inspect the mesh for errors and correct them using the Photoshop "Erase" and "Pencil" tools (width of about 6 pixels). If you are unsure of a particular grainboundary location, try turning on the visibility of the other RGB image at the base of the stack. Boundaries that are obscure in one view may be obvious in the other view. This inspection is the most time-consuming part of the image processing, but it provides the analyst with an excellent opportunity to scrutinize the image carefully and to record any observations in a new "comments" layer at the top of the RGB stack. Circles around unusual or uninterpretable features in the RGB image, text comments, or arrows pointing to good examples of phenomena of interest are all candidates for the "comments" layer.

- Erase the margins of the mesh back to the first complete grain all the way around the edges of the mesh image. When you're satisfied with the result, copy and paste this final coarse mesh into a new layer at the top of the edge-stack file and name the layer "Final Coarse Mesh." If necessary, reposition it to coincide with the starting coarse mesh layer below it.
- In order to continue with the next steps in the analysis, the background of the final coarse mesh layer needs to be reset to white (at this point it is transparent). Do this by creating a new layer just below the final coarse mesh layer and setting the fill color to white. Then merge the two layers. Fill the background of the "Final Coarse Mesh" layer with white and rethreshold it to ensure that the only color values in this layer are black and white. The correct final configuration will show only a black edited grain boundary (the mesh) and a white background.

\subsubsection{Skeletonize the Corrected Grain-Boundary Mesh}

- Duplicate the "Final Coarse Mesh" layer into a new layer above.

- With this new layer selected, invoke Filter $>$ IP• Morphology $>$ Pruned skeleton followed by Filter $>$ IP•Morphology $>$ Thicken skeleton and rethreshold the image, accepting everything that is not white (first make sure the foreground color in Photoshop is black and the background color is white!). These operations will reduce the coarse mesh to 1-pixel width, trim off most dangling pixels, and ensure that all pixels in the resulting mesh are 4-connected (share at least one edge with another signal pixel).

- As a final step, ensure in this final fine-mesh image that there are no stray pixels or open endpoints. This final check is best done at a magnification factor of 200 in Photoshop. In addition, make sure the background continues all the way to the edge of the canvas.

- Invert the final fine mesh layer so that the grain boundary is white and the background is black.

- To complete your RGB stack, copy and paste this final fine mesh back into a new layer at the top of the RGB stack. You may need to position it into coincidence above the coarse mesh image. Save and close the RGB stack. This step completes the image-processing portion of the analysis.

\section{Image Analysis}

In this phase of the process, numerical data are generated from the fine mesh grain-boundary image. Two families of measurements are compiled: global measurements that pertain to the image in its entirety, and discrete measurements that are generated for each grain.

FoveaPro algorithms assume a value of 0 (black) for signal and 255 (white) for nonsignal. In this scheme, grain areas must be black and grain boundaries must be white. Invert the mesh image if necessary to achieve this relation. (Image $>$ Adjustments $>$ Invert), and ensure that your Photoshop 
foreground color is black and background is white. Set up a $\log$ file using Filter $>$ IP•Utilities $>$ Setup Log File. This file will capture the output of the analytic routines.

\subsection{Global Measurements}

Record these measurements for each analysis either through the FoveaPro log file or screen captures.

\subsubsection{Mean Planimetric Grain Area}

Determine the mean planimetric grain area by dividing the total sample area by the number of grains. This value provides a double check on statistics generated from the grainspecific measurements.

- Ensure that the spatial calibration in Photoshop is correct.

- Select the Photoshop magic wand tool, set the color tolerance to 1, and check the "Contiguous" checkbox in the brush controls panel. Ensure that the Photoshop foreground color is set to black.

- Select a spot on the image between the outer edge of the grain-boundary mesh and the outside edge of the image. If the spot is selected correctly, dotted region boundaries will appear along the outer perimeter of the image area and along the outer edge of the sample (fig. 9).

- Invert the selection (Select>Inverse). If the inversion is correct, the dotted boundary around the outer edge of the image will disappear.

- On the Photoshop menu bar choose Analysis>Record Measurements. A window will open along the bottom of the screen with one or more measurements in it. Verify that the scale factor is correct and record the "Area" entry. The units should be square centimeters $\left(\mathrm{cm}^{2}\right)$. Check that the number is the correct order of magnitude for the approximate sample area. (This is a common-sense check.)

- As a quick check on the integrity of the final fine mesh file, invoke Filter $>$ IP Measure Features $>$ Count. This final screen will return a value for the number of grains (n) in the image that is one greater than the correct value.

- Record the Photoshop scale factor, uncorrected count, and sample area.

- Divide the sample area by $n-1$. Record this value as the planimetric mean grain area.

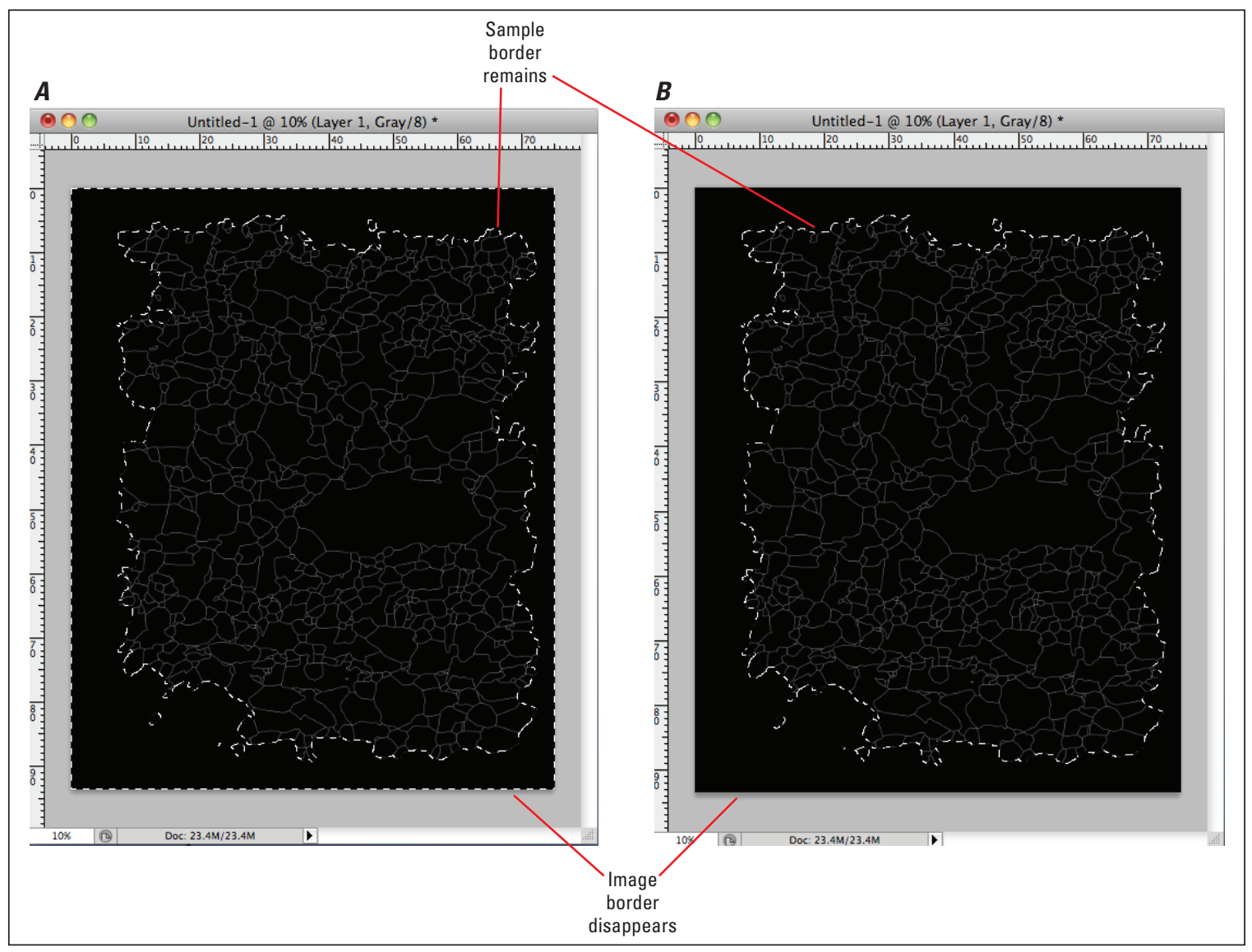

Figure 9. Determining mean planimetric grain size by finding total grain area and dividing by the number of grains. $A$, Selection of image area outside of the grain. $B$, Inversion to select only the area occupied by grains. The dotted line around the perimeter of the image will disappear when the selection is inverted. 


\subsubsection{Distribution Plots}

As a first cut, some of the display tools in FoveaPro can be used to plot distributions and relations (such as grain area, aspect ratio, or moment angle). A few suggestions are given below. Note that generating these plots in FoveaPro is timeconsuming because this program recalculates all the needed values from the image.

- Select Filter $>\mathrm{IP} \bullet$ Measure Features $>$ Plot (Distribution) and select any parameter for which you would like to see a histogram distribution of up to 30 bins. The window that displays the histogram also provides the statistics. You can save the bin data and the image to the log file. Typically, a user saves distribution plots for area, elongation, equivalent diameter, and moment angle. These routines will also return values for $n$, the number of grains in the measurement. Check the value of $n$ generated using the FoveaPro count command described in the second paragraph above against the total number of grains displayed at the top of the distribution plot (fig. 10). They should be the same.

\subsubsection{Scatter Plots}

- Select Filters $>$ IP•Measure Features $>$ Plot (Scatter) and select $\mathrm{x}$ and $\mathrm{y}$ values for a simple scatter plot. Save scatter plots for area compared with elongation and moment angle compared with area (fig. 11).

\subsubsection{Cluster Analysis}

- Other available operators of interest include Filter $>$ IP•Measure Global $>$ Clustering, which performs a nearest-neighbor distance analysis and compares the values to a random distribution. This option also creates a rose plot (fig. 12).

\subsection{Grain-Specific Measurements}

The final step of the image analysis phase is the generation of the master data table, which calculates a suite of parameters for every grain in the image. Each grain is assigned a unique number during this step; for identification purposes these numbers can be plotted onto the final fine grain-boundary mesh image at the top of the edge stack.

\subsubsection{Generate the Master Data Table}

- Ensure that Photoshop's foreground color is set to black.

- With the final fine grain-boundary mesh layer selected in the edge stack, invoke Filter $>$ IP $\bullet$ Measure Features $>$ Measure All Features. Select "Create New File" and name the output file with the core and sample designation leaving the "Feature Data" portion of the filename intact (see "File Naming Conventions" in

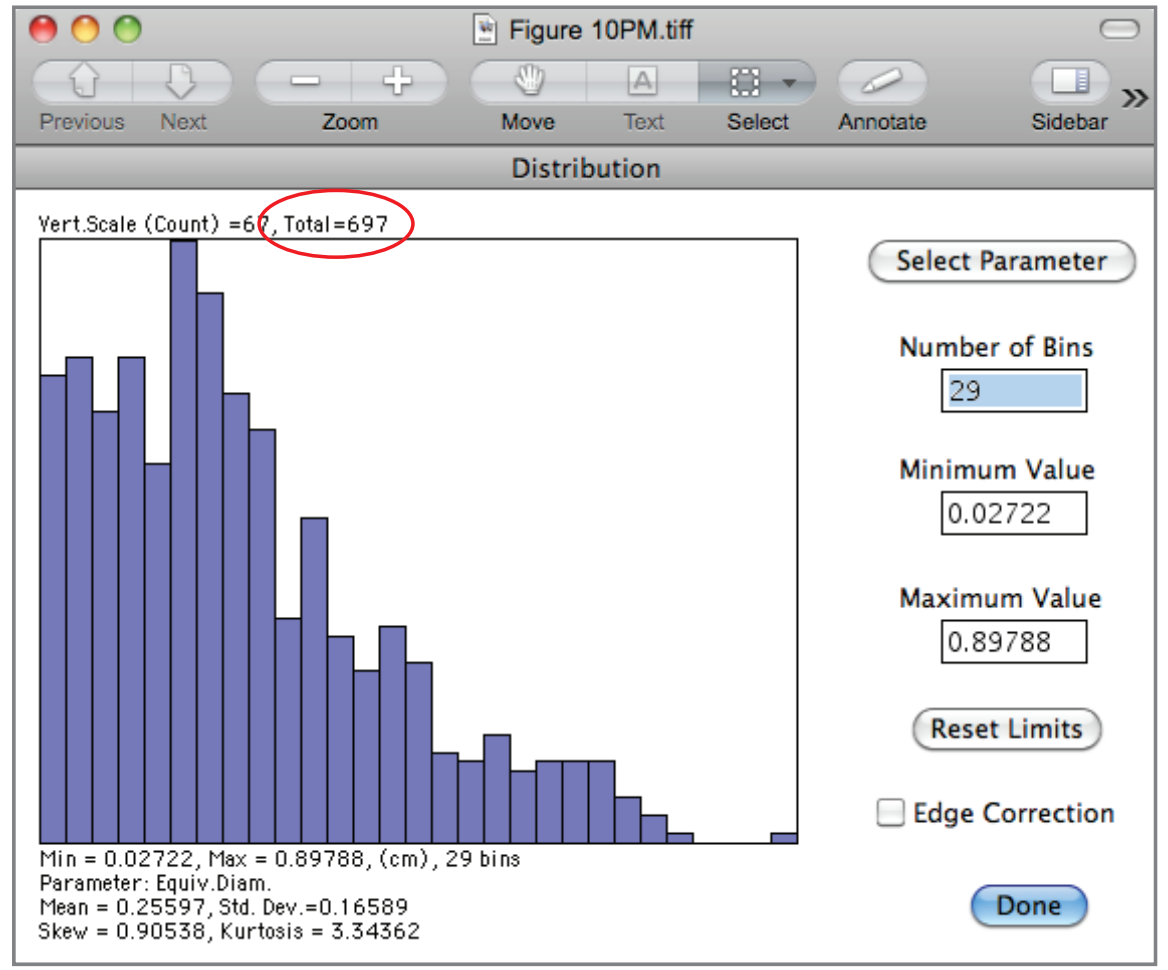

Figure 10. A FoveaPro distribution diagram based on equivalent diameter. The total number of grains counted by this algorithm (red circle in figure) should be 1 less than the number detected by the global "count" command. The nature of the population will be apparent from these types of plots. 


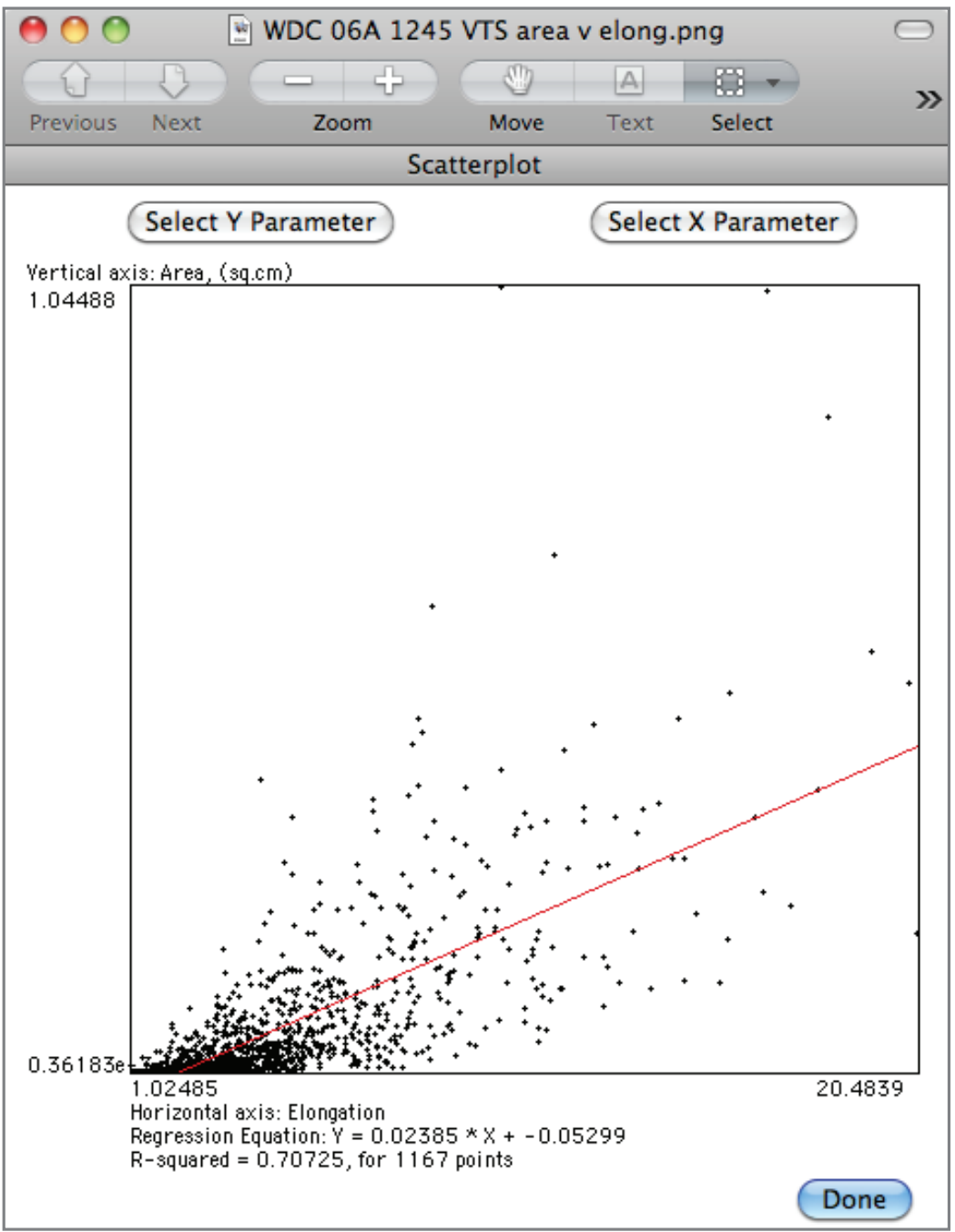

Figure 11. FoveaPro scatter diagram of points. Elongation on the $x$-axis is compared with area on the $y$-axis; a linear fit to the data is superimposed.

appendix 2.) These steps invoke the primary set of FoveaPro measurement algorithms and will produce a large, space-delimited plain-text data table that provides information for more than 40 parameters for each grain. If you have a large number of grains in the image (more than 1,000$)$ it is likely to take some time to execute. Save the output file and then import it into Excel and save it. For a description of how these parameters are computed, please refer to Russ (2011a).

- The Measure All Features filter generates data table entries that are not used for further analysis. Delete these columns from your formatted Excel data sheet before continuing with the further analysis. These columns are

- O Num_Holes

- V Hole Fraction

- Z Skel.End_Points through AQ Integ.Optic. Dens. BB Min.Separation_Dist through BE Adjusted_Count

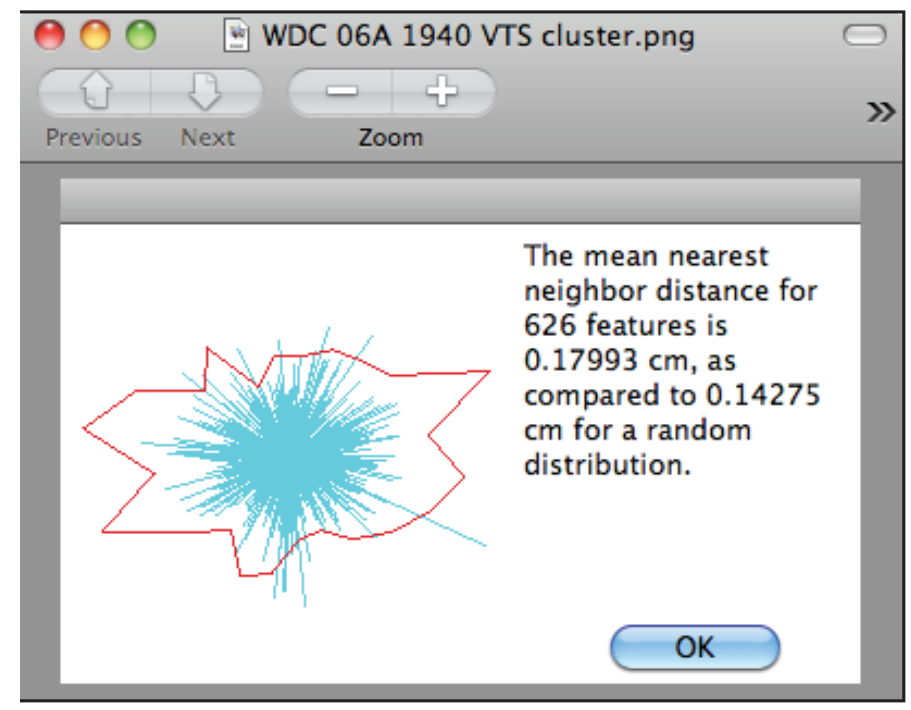

Figure 12. FoveaPro cluster-analysis plot. This type of diagram gives a quick impression of grain elongation and alignment. 


\subsubsection{Statistical Measurements}

Full statistics can now be generated on the grain population for each of the measured parameters. In a Windows environment, the required statistical routines can be accessed directly from Excel. In the Mac OS environment, these routines are an add-on to Excel (StatPlus:Mac) that must be downloaded and installed. Instructions given here refer to the Mac OS environment.

- With the Excel data spreadsheet open, launch StatPlus.

- Select Statistics $>$ Basic Statistics and Tables $>$ Descriptive Statistics. When queried for the columns for which the statistics will be computed, select all data columns in the Excel spreadsheet except the first one (which contains the grain identification numbers). Select "Advanced Options" from the Descriptive Statistics window and check the "Plot histogram" and "Overlay histogram" boxes; then enter 100 in the box opposite "Number of intervals" (fig. 13).

- After the stat file completes execution, save it and name it with the core and sample identification number and the word "stats" just ahead of the .xlxs extension.

- Check the first entry for "Count" under the "Area" parameter located in cell B3. The value displayed here will be 1 less than the value for the number of grains you retrieved from Measure Features $>$ Count in section 3.1.1. If this is not the case, then an undiagnosed problem remains in the fine mesh and you need to go back and correct it. (For instance, if the statistical count is higher than the correct value, stray single pixels probably exist in the fine mesh image; if the value is lower than the correct value, then an open edge probably lies somewhere in the image.)

- After verifying that the feature count is correct, retrieve the mean and median values for area, equivalent diameter, aspect ratio and form factor from the stats file and record them in a data table to facilitate post-processing analysis.

\section{Postprocessing}

Once the grain-level data tables have been acquired and the descriptive statistics have been generated for all samples, the postprocessing phase of the analysis can be carried out. This is the phase that uses the results of the analyses to address scientific questions, and it is typically initiated by plotting and examining the behavior of specific parameters as a function of depth or age and temperature within the ice body and comparing the results with those from other sites and with other data sets from the same site. Some behaviors are expected and can be observed in most deep ice cores; some observations may be unique to the site. It is important to remember that, unlike deep sea-sediment cores which number in the thousands, relatively few deep ice cores have ever been retrieved, so the basis for comparison from site to site is limited. Several examples are provided here to show how the data generated from the digital imagery of an ice core can be used to investigate the textural, stratigraphic, and structural characteristics of an ice sheet.

\subsection{Example 1. Mean Grain Size as a General Guide}

Although the mean grain size is not particularly informative in anything other than a general sense (grain-size distributions are typically log normal, not Gaussian), examination of mean grain area as a function of depth or age can be used to quickly identify important changes in chemistry, particulate loading, and ice temperature. Grain size changes can result from the deposition of aerosols from nearby minor volcanic eruptions or distant major eruptions; increased dust deposition associated with glacial periods (resulting from increased availability and atmospheric transport during glacial intervals); and increasingly warm temperature in samples that are increasingly close to the bed of the glacier. Because the majority of early published literature also typically characterized grain-size behavior by mean grain area, mean intercept length, or mean grain diameter, comparison with these earlier works requires the continued use of mean grain size.

Mean grain areas of the West Antarctic Ice Sheet Divide (Antarctica) deep ice core (WDC 06A) between 120 and 3,400 meters $(\mathrm{m})$ were determined on a 20 -m sampling interval (fig. 14). Although the pronounced ' $M$ ' shape to the curve above $2,500 \mathrm{~m}$ depth is unique to this core, these data have several features in common with cores from other sites. The steep increase in mean grain area with depth from the shallowest sample to about $500 \mathrm{~m}$ is typical of the "normal grain growth" regime observed in many ice sheets, as are the general grain size depression during and around the Last Glacial Maximum and the extreme coarsening as the temperature of the ice approaches its pressure melting point near the bed. The abrupt, narrow drop in mean grain size at the Antarctic Cold Reversal (the depth of which has been verified by stable isotope analysis) has been observed in other Antarctic cores as well.

The depth and age of these types of transitions in grain size should correlate with other measurements that are made on the core, such as chemistry, particle count, electrical properties, and possibly stable isotopes.

\subsection{Example 2. Population Statistics and Distributions}

Characterization of population distributions is more informative than examination of the simple mean, which can be strongly influenced by the presence of a few large grains. Grain populations can be compared on the basis of actual measured sizes, or the populations can be normalized to their mean or mode for comparison. Segregation of specific populations (such as the largest 5 percent of grains in a sample) is another way to investigate the effects of grain growth and recrystallization.

A quantile-quantile comparison was compiled of three samples from before, within, and after the Antarctic Cold Reversal (ACR) at West Antarctic Ice Sheet Divide as identified from the stable isotope record (fig. 15). The comparison shows that the difference in mean grain size 


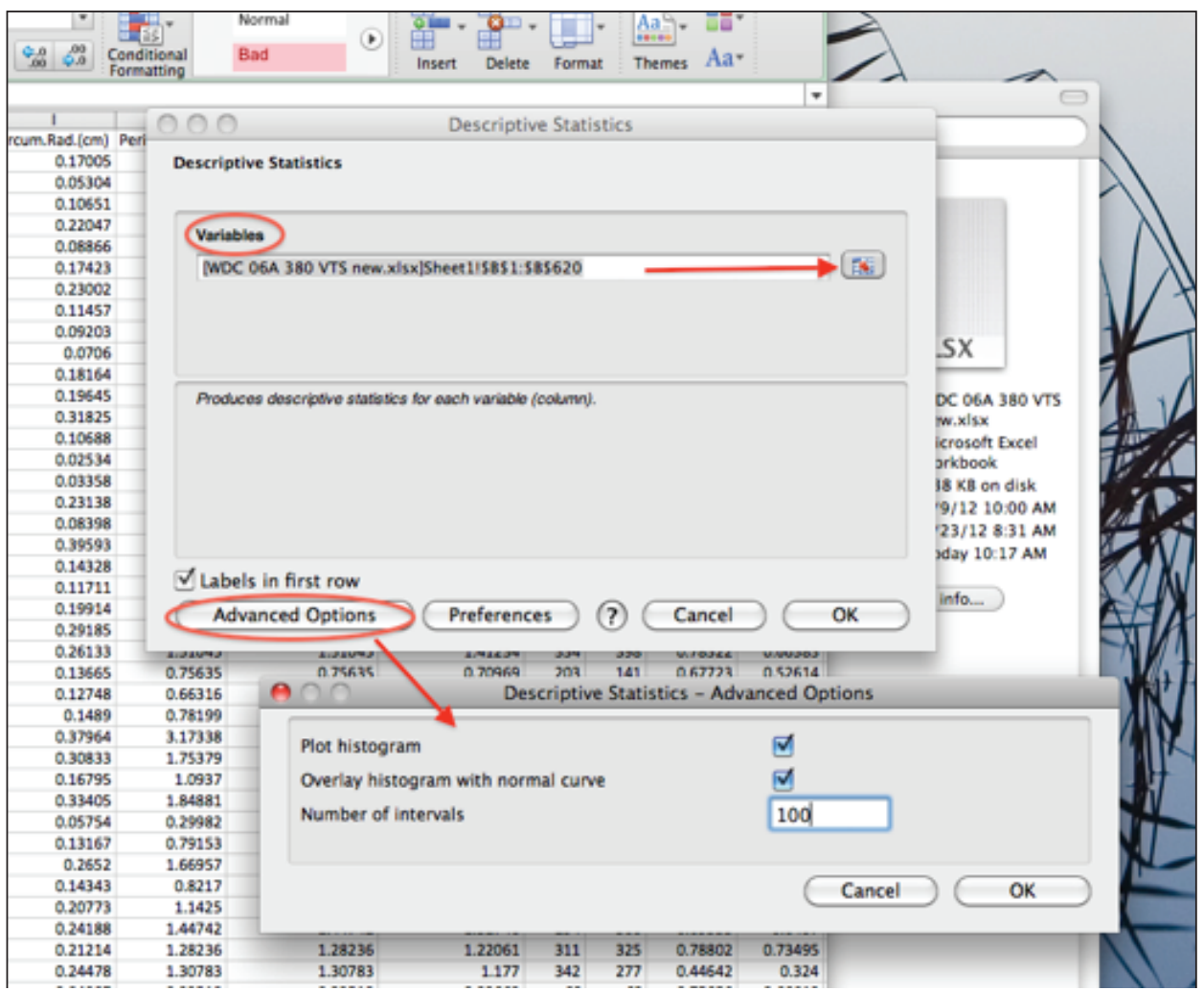

Figure 13. StatPlus, “Descriptive Statistics" window.

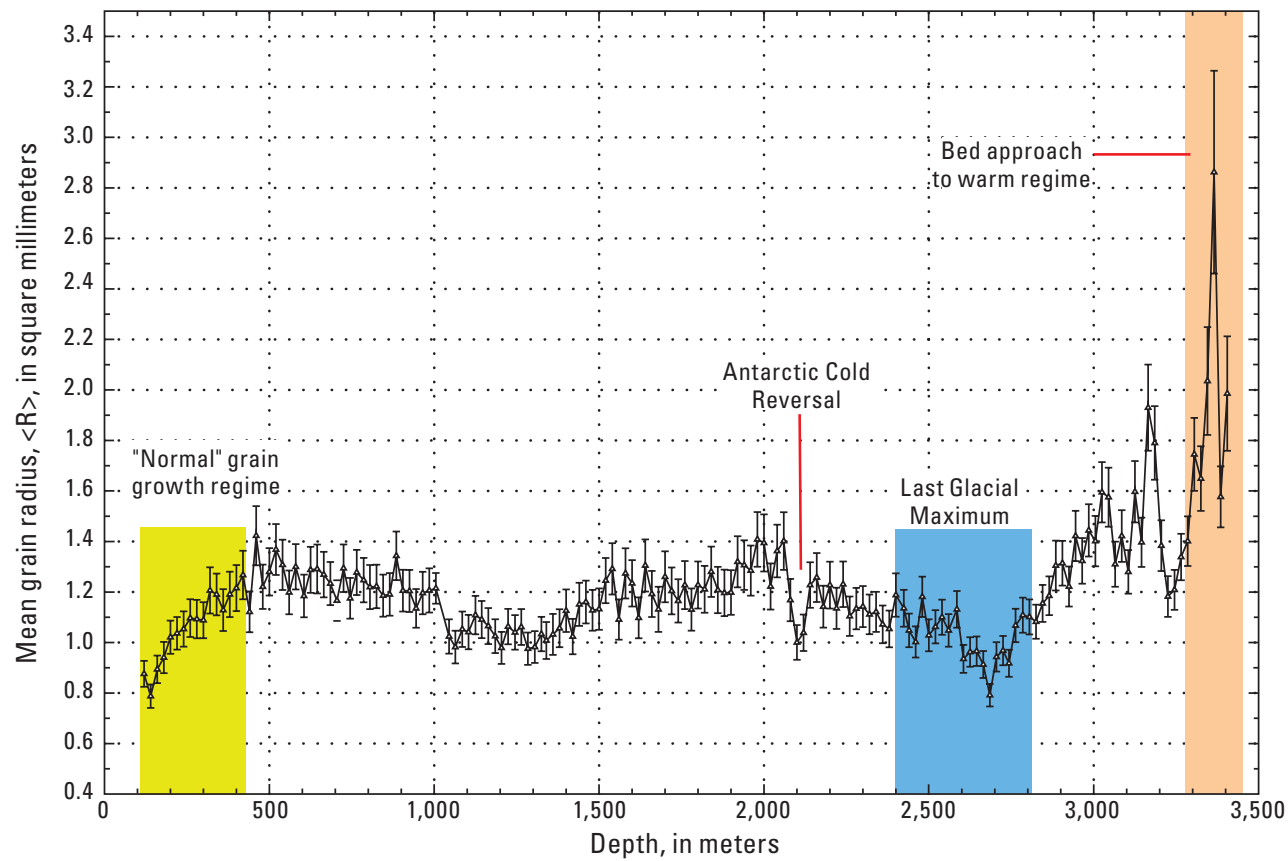

Figure 14. Mean grain radius as a function of depth in the West Antarctic Ice Sheet Project main core (WDC 06A). Core-sample interval, 20 meters. Features of this dataset that are similar to those in other Antarctic cores include the following: steep initial curve marking grain growth in the "normal" grain-growth regime); a narrow minimum at the Antarctic Cold Reversal (about 2,100 meters depth); a region of depressed grain size during and around the time of the Last Glacial Maximum; and extreme grain coarsening in the warm-temperature regime at the bottom of the ice sheet, where the in-place temperature is warmer than $-10^{\circ} \mathrm{C}$. 


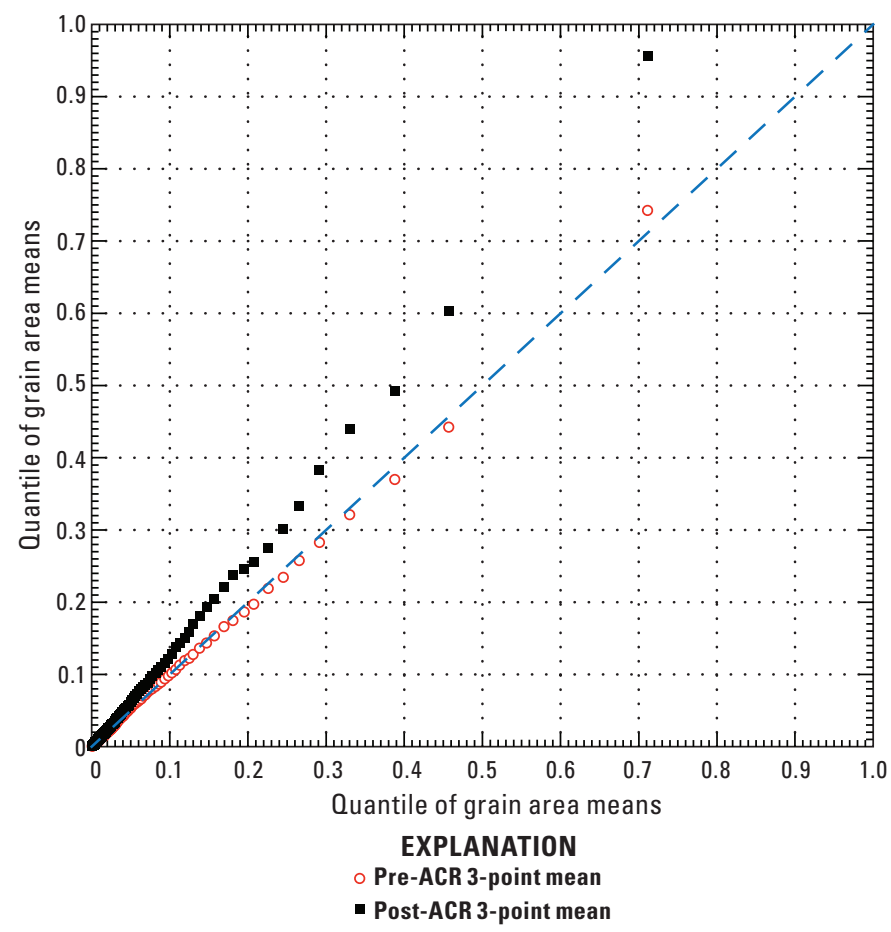

between within-ACR and after-ACR samples that is seen in figure 14 originates primarily from the coarsest 15 percent of the grain population in the after-ACR sample; the remaining 85 percent of the grain population smaller than this coarsest part is very similar in size distribution. The entire populations of the before-ACR and within-ACR sample are essentially identical. A probable explanation resides in the dust record, which uses cerium as a continental-dust proxy (J.R. McConnell, pers. commun., 2013). The dust record, in combination with the isotopic record, indicates that although
Figure 15. Three grain-area populations from the West Antarctic Ice Sheet Divide core before, during, and after the Antarctic Cold Reversal (ACR). Values on $\mathrm{x}$-axis derived from samples that originated during the Antarctic Cold Reversal. Values on y-axis represent the difference in grain sizes in each 1 percent quantile between ice from the ACR (depth 2,040 to 2,180 meters) and ice either older than the ACR (depth 2,200 to 2,240 meters) or younger than the ACR (depth 1,980 to 2,020 meters). The entire population distribution was nearly identical just before and during the ACR. Mean grain size increased in younger ice; this increase is attributable to the largest 15 percent of grains in that ice. This relation suggests a growth-limiting factor in ACR and older ice that is absent in younger ice.

the temperature was warmer in the time immediately before the ACR, dust concentrations were still higher than after the $\mathrm{ACR}$ and that this higher dust concentration is the likely factor that limited grain growth before ACR time.

\subsection{Example 3. Ice Behavior Compared}

It is useful to compare the grain characteristics among ice cores from different sites in order to separate behaviors that are common to all ice sheets from those that may be unique to the ice dynamics at a particular site. For example, rates of grain growth in the "normal" grain growth regime from several ice core sites in Antarctica and Greenland are compared in figure 16. The newly measured growth rate at West Antarctic Ice Sheet Divide (red triangle in fig. 16) is commensurate with previous observations and falls near the fitted curve expressing the temperature dependence of this growth rate in the uppermost portions of an ice sheet. (Other data compiled by Cuffey and Patterson, 2010.)

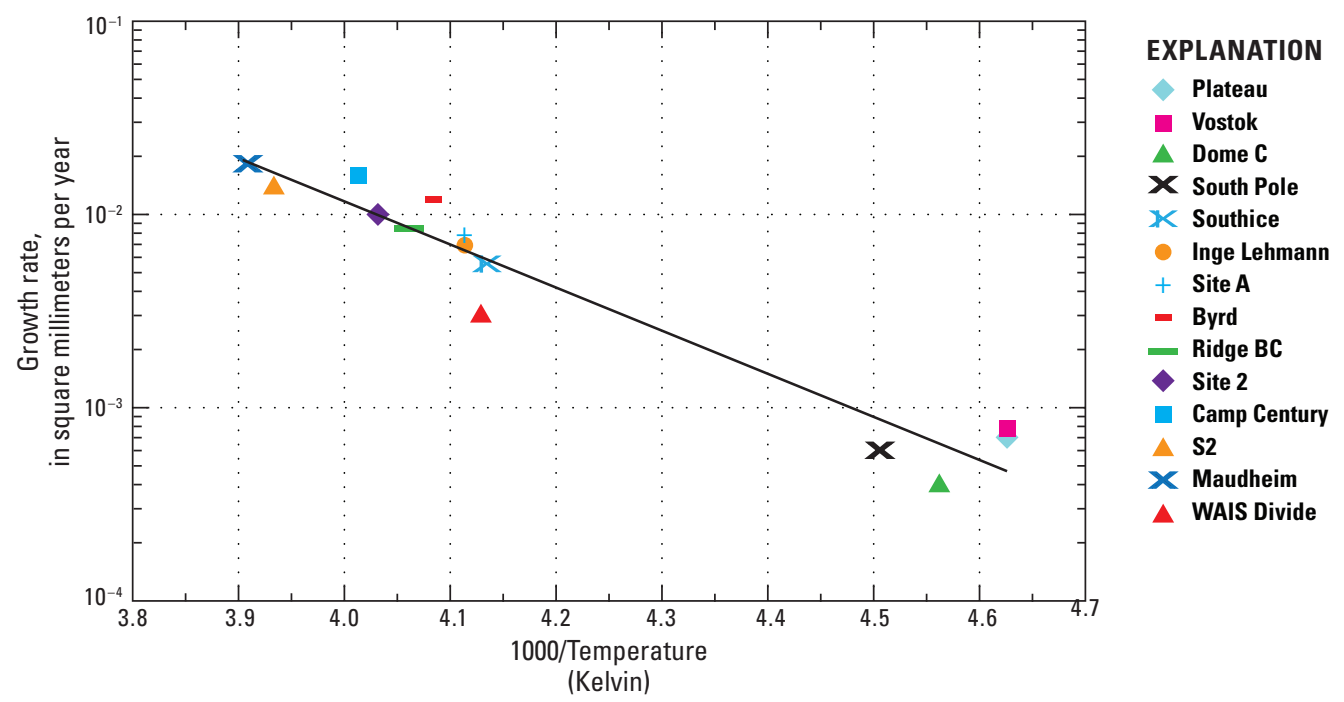

Figure 16. Rates of recrystallization in a "normal" grain-growth regime from 14 ice-core sites in Antarctica and Greenland. The growth rate at West Antarctic Ice Sheet Divide is commensurate with previous observations, and it falls near the fitted curve that expresses the temperature dependence of growth rate in the uppermost portions of an ice sheet. (Modified from Cuffey and Paterson, 2010.) 


\section{Summary}

Image processing and analysis are powerful tools for extracting quantitative information from digitally stored images. This report provides step-by-step guidance for manipulating digital images of a specific substance, glacier ice, with applications in the fields of glaciology and paleoclimatology. In the broader field of image interpretation, these types of techniques can be used in applications as diverse as metallurgy, forensics, and quality control in manufacturing.

As with any set of tools, there are many ways to accomplish the same task. The instructions given here have proven to be generally successful for the task of extracting size, shape, and texture information from samples of glacier ice, but in specific instances, different approaches may sometimes prove to be more successful. As an analyst gains experience with the processing and analysis algorithms used here, other choices should be explored to gain familiarity with other algorithms and their potential applicability to a broader range of image types.

Two cautionary notes: first, it is imperative that the analyst always understands the nature of each image-processing operator and each analytical algorithm used at the array or matrix level and not only at the image level. Using an operator or algorithm that is poorly understood at the computational level can lead to inappropriate applications of such operators or algorithms. So doing may yield undetected incorrect results.
Finally, always document each step in the processing and analysis procedures. This practice serves several purposes-it allows the analyst to recreate an analytical pathway long after the recollection of how a particular image was treated has faded and, most important, it provides a chain of analytical integrity that is necessary for robust scientific research. In short-always know what you are doing and always document what you have done.

\section{References Cited and Recommended Reading}

Cuffey, K.M., and Paterson, W.S.B., 2010, The physics of glaciers (4th ed.): Amsterdam, Elsevier, 693 p.

O'Gorman, Lawrence., Sammon, M.J., and Seul, M., 2008, Practical algorithms for image analysis (2d ed.): New York, Cambridge University Press, 349 p.

Russ, J. Chris, 2011, Fovea Pro 4.0 Users' Manual. Bundled with Fovea Pro Distribution CD-ROM: Raleigh, N. Car., Reindeer Graphics.

Russ, John C., 2011a, The Image Processing Handbook (6th ed.): Boca Raton, Florida, CRC Press, 771 p.

Russ, John C., 2011b, The Image Processing Cookbook (2d ed.): Scotts Valley, Calif., Amazon Createspace, 138 p. 

Appendixes 



\section{Appendix 1. Image-Processing Walk Through}

1. Remap the image to achieve the best possible contrast

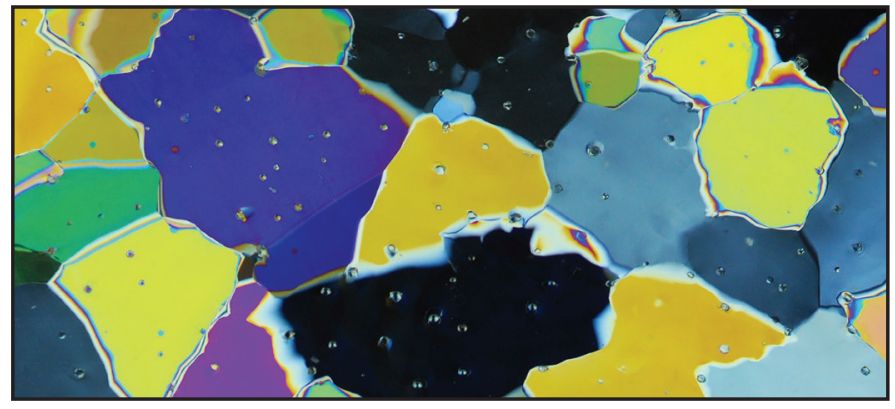

Original Red-Green-Blue (RGB) image

2. Locate the edges

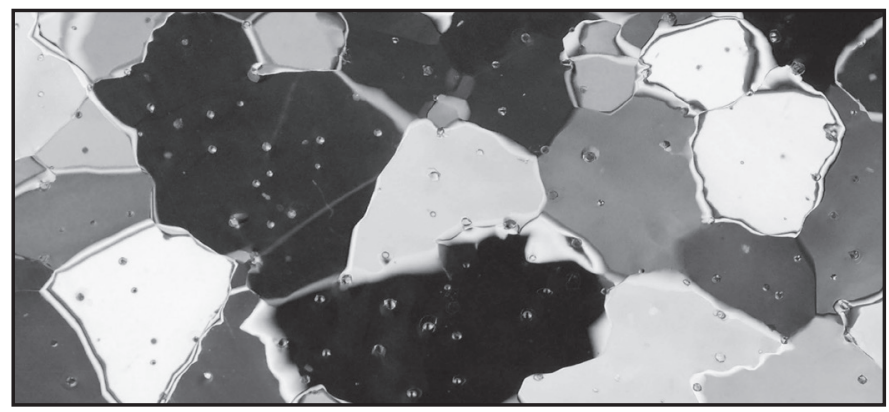

Most significant channel of the Principal Components Analysis (PCA)

3. Binarize (threshold) the edge file

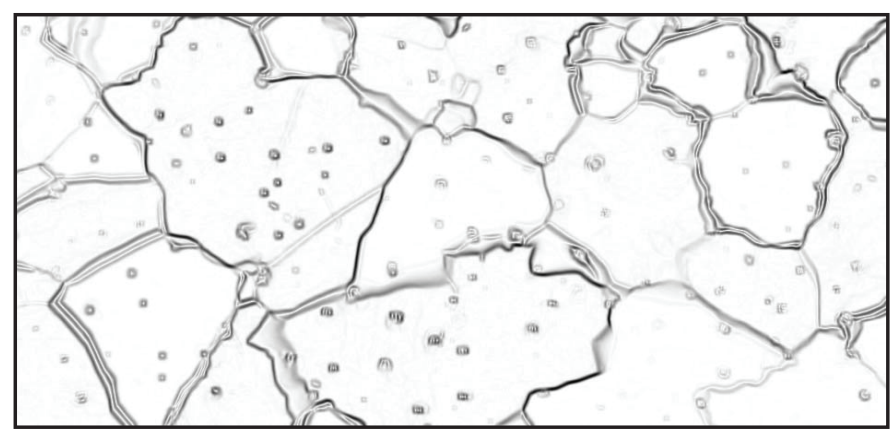

$3 \mathrm{~d}$ order gradient vector edges

4. Reject unwanted features, clean up edges

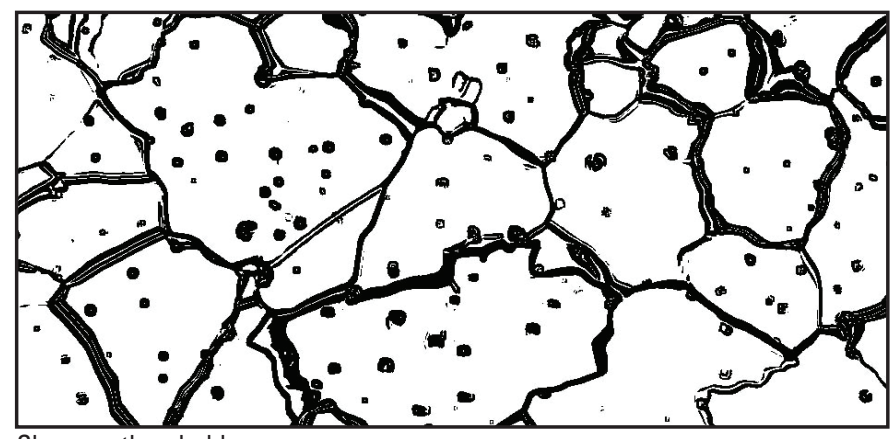

Shannon threshold

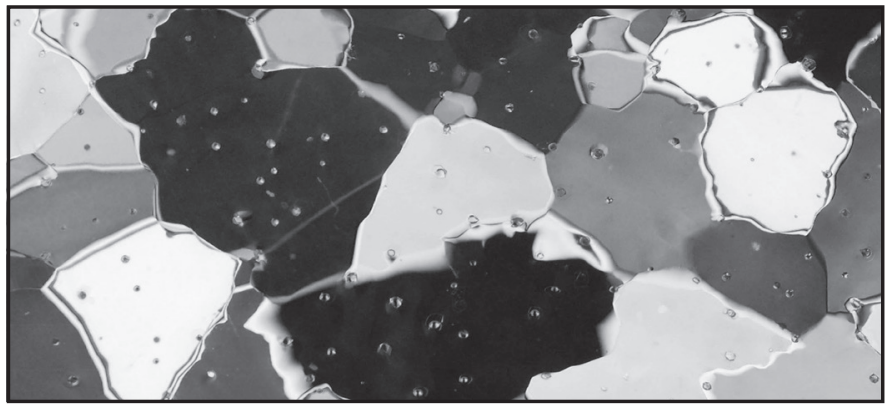

Most significant channel of the Principal Components Analysis (PCA)

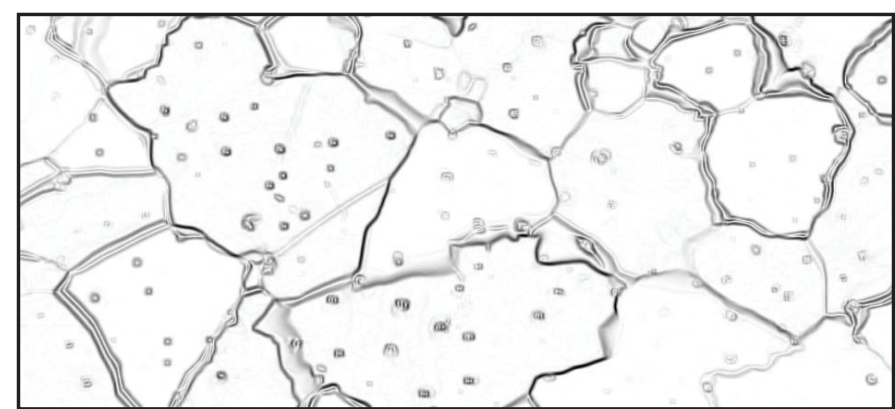

$3 \mathrm{~d}$ order gradient edges

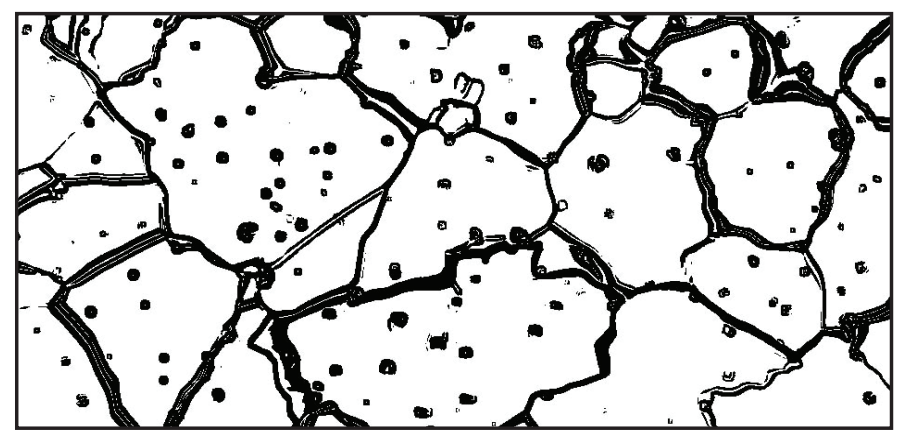

Shannon threshold

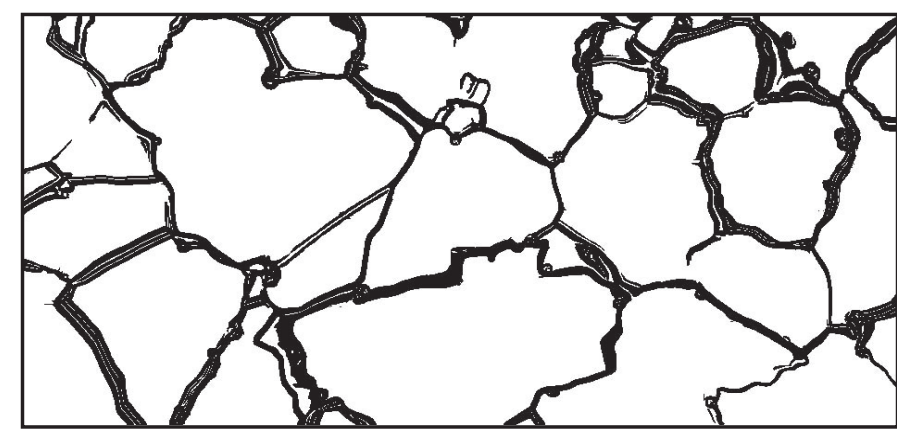

Reject features 
5. Use morphological operators to improve the continuity of the edge mesh

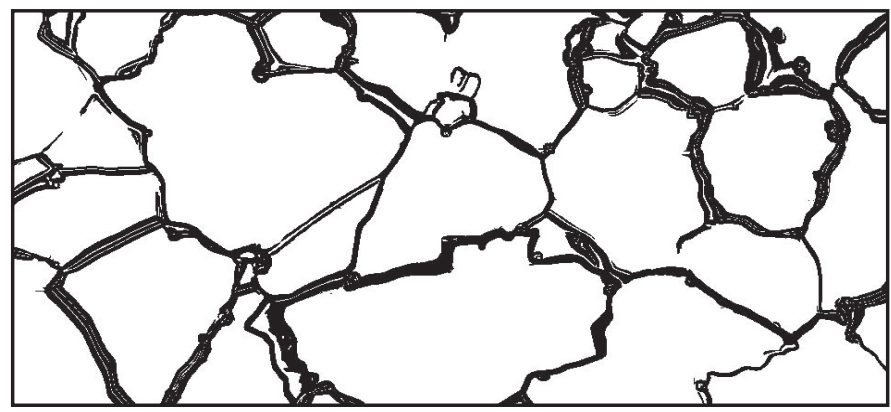

Reject unwanted features

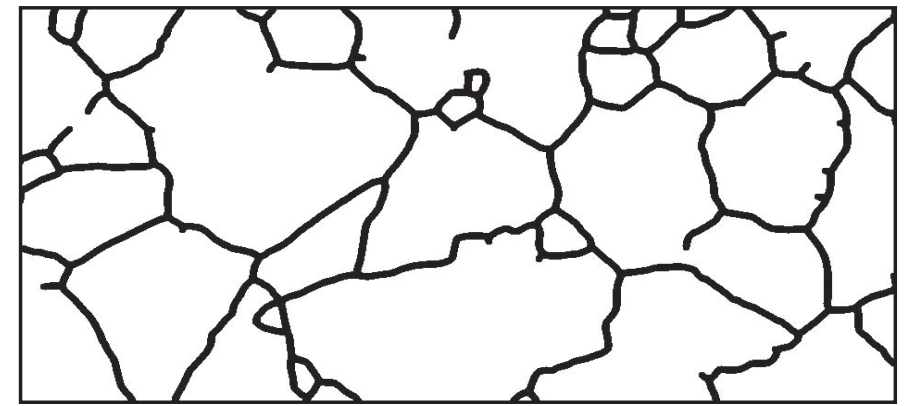

Euclidean Distance Mapping (EDM) morphological operators produce the starting coarse edge mesh.

6. Superimpose the coarse edge mesh back onto the RGB image and check/correct it.

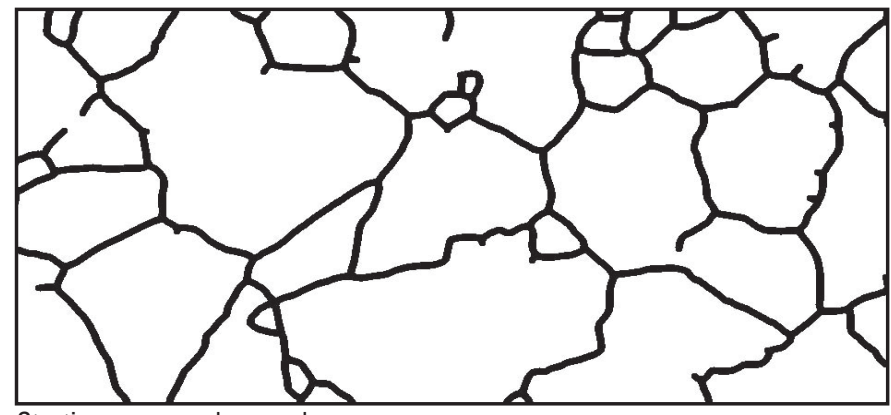

Starting coarse edge mesh

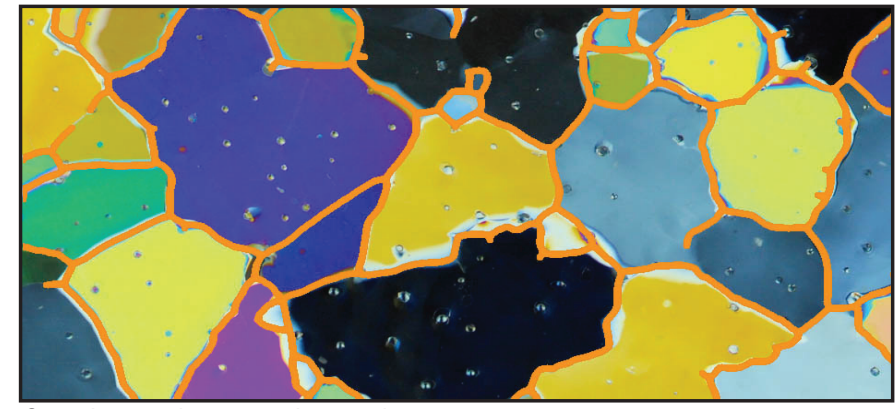

Superimposed coarse edge mesh

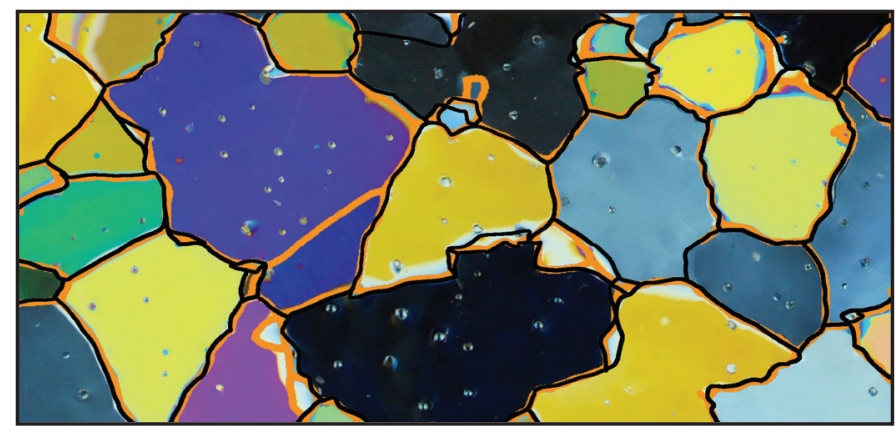

Edited coarse edge mesh

7. Skeletonize and thicken the corrected edge mesh

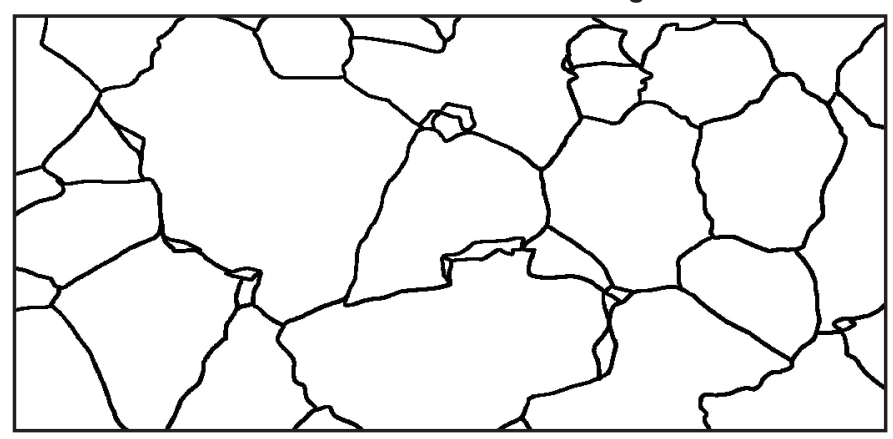

Extracted, edited coarse edge mesh

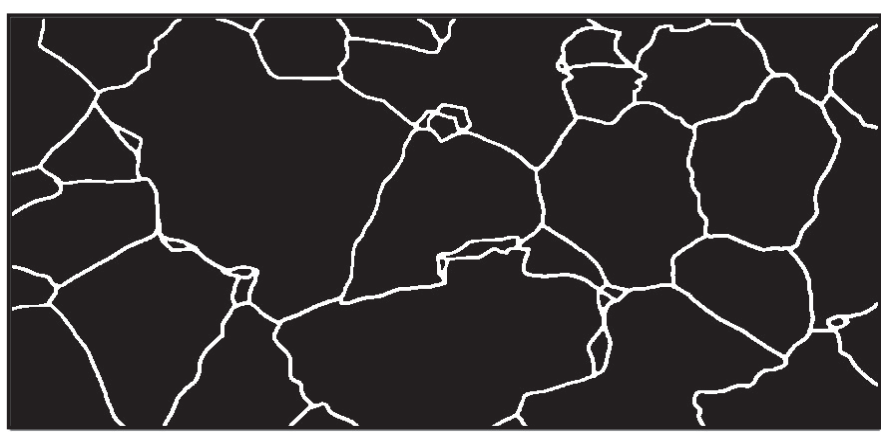

Skeletonized, thickened, inverted fine edge mesh ready for analysis 


\section{Appendix 2. File Naming Conventions}

\section{File naming conventions-in order of creation during analysis}

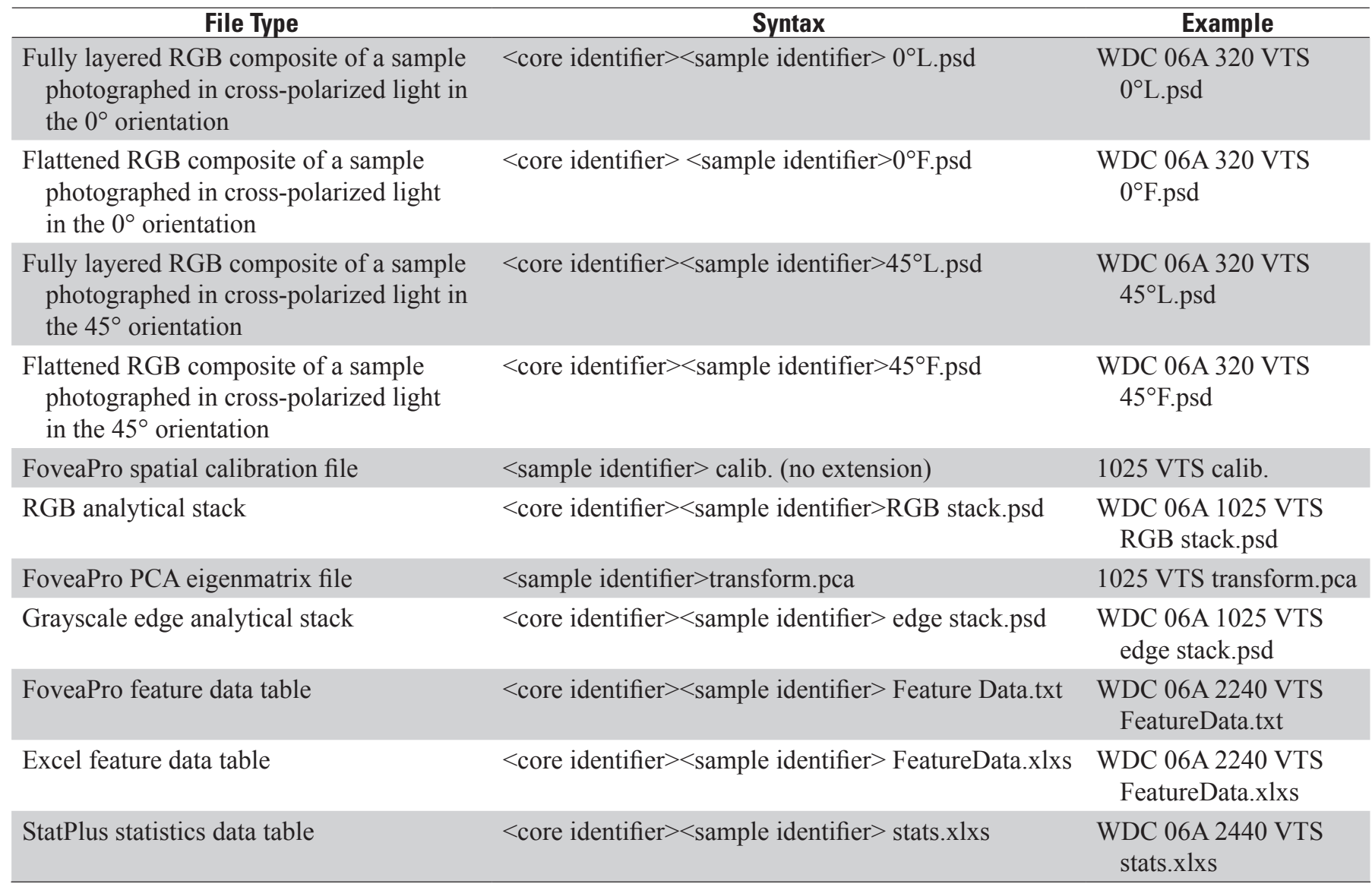

Publishing support provided by:

Denver Publishing Service Center, Denver, Colorado

For more information concerning this publication, contact:

Center Director, USGS Geosciences and Environmental Change Science Center Box 25046, Mail Stop 980

Denver, C0 80225

(303) 236-5344

Or visit the Geosciences and Environmental Change Science Center Web site at: http://gec.cr.usgs.gov/

This publication is available online at:

http://dx.doi.org/10.3133/tm7D1 


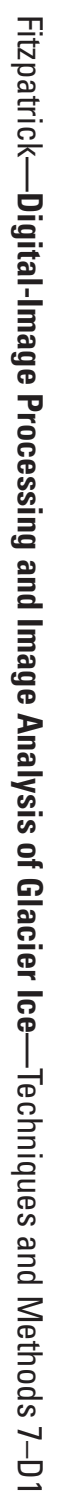

